\title{
Higgs boson decays to dark photons through the vectorized lepton portal
}

\author{
Qianshu Lu, ${ }^{a, b}$ David E. Morrissey ${ }^{a}$ and Alexander M. Wijangco ${ }^{a}$ \\ a TRIUMF, \\ 4004 Wesbrook Mall, Vancouver, BC, V6T $2 A 3$ Canada \\ ${ }^{b}$ Division of Engineering Science, University of Toronto, \\ Toronto, ON, M5S 1A7 Canada \\ E-mail: qianshu.lu@mail.utoronto.ca, dmorri@triumf.ca, \\ awijangco@triumf.ca
}

ABSTRACT: Vector-like fermions charged under both the Standard Model and a new dark gauge group arise in many theories of new physics. If these fermions include an electroweak doublet and singlet with equal dark charges, they can potentially connect to the Higgs field through a Yukawa coupling in analogy to the standard neutrino portal. With such a coupling, fermion loops generate exotic decays of the Higgs boson to one or more dark vector bosons. In this work we study a minimal realization of this scenario with an Abelian dark group. We investigate the potential new Higgs decays modes, we compute their rates, and we study the prospects for observing them at the Large Hadron Collider and beyond given the other experimental constraints on the theory. We also discuss extensions of the theory to non-Abelian dark groups.

Keywords: Beyond Standard Model, Higgs Physics

ARXIV EPRINT: 1705.08896 


\section{Contents}

1 Introduction 1

2 Fields, masses, and interactions 3

2.1 Minimal masses and interactions 3

2.2 Additional interactions 5

3 Higgs boson decays to dark vectors $\quad 6$

$\begin{array}{ll}3.1 & \text { Higgs branching fractions }\end{array}$

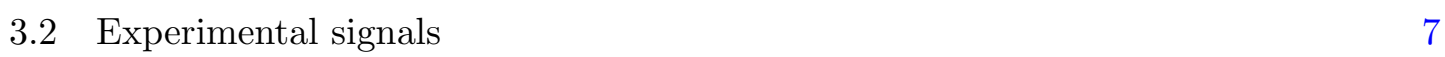

4 Precision electroweak and collider constraints $\quad 8$

4.1 Electroweak constraints 8

4.2 Collider bounds 9

$\begin{array}{lll}4.3 & \text { Higgs stability } & 11\end{array}$

5 Connections to dark matter $\quad 12$

$\begin{array}{lll}5.1 & \text { Relic densities } & 12\end{array}$

$\begin{array}{lll}5.2 & \text { Direct detection } & 13\end{array}$

$\begin{array}{lll}5.3 & \text { Beyond the minimal scenario } & 14\end{array}$

6 Comments on the non-Abelian case $\quad \mathbf{1 5}$

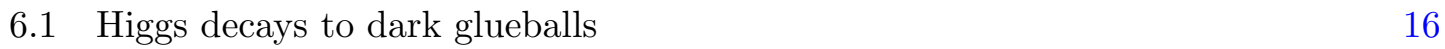

$\begin{array}{lll}6.2 \text { Constraints } & 17\end{array}$

$\begin{array}{lll}\text { 6.3 Dark matter considerations } & 18\end{array}$

$\begin{array}{lll}7 \text { Conclusions } & 19\end{array}$

$\begin{array}{lr}\text { A Higgs loop functions } & 19\end{array}$

$\begin{array}{lr}\text { B Electroweak self-energies } & 21\end{array}$

C Renormalization group equations $\quad 22$

\section{Introduction}

Dark sectors have been studied extensively in recent years [1-5]. Such sectors consist of new states that interact only very weakly with the Standard Model (SM). This allows the new physics in the dark sector to be relatively light, with characteristic mass well below the electroweak scale, while still being consistent with current experimental tests. Dark sectors may also be related to (or comprise) the dark matter in the universe [6-9]. 
While the range of possibilities for dark sectors is enormous, particular attention has been given to those that connect to the SM through a set of portal operators:

$$
\begin{aligned}
\text { Vector Portal : } & \frac{\epsilon}{2} B_{\mu \nu} X^{\mu \nu} \\
\text { Higgs Portal : } & \left(A \phi+\kappa \phi^{2}\right)|H|^{2} \\
\text { Neutrino Portal : } & y_{N} \bar{L} \widetilde{H} N
\end{aligned}
$$

First, in the vector portal, a new Abelian vector boson $X$ couples to the SM through kinetic mixing with hypercharge [10, 11]. Second, in the Higgs portal [12, 13], a new scalar connects with the SM Higgs field. And third, in the neutrino portal a new gauge singlet fermion $N$ connects to the SM lepton and Higgs doublets. These portals represent the three ways in which a new field with no SM charges can couple to the SM at the renormalizable level. As such, these interactions are non-decoupling, and the most sensitive searches for light new physics connecting to us through these interactions are typically lower-energy experiments with very high intensity or precision [4].

The portal interactions of eqs. (1.1), (1.2), (1.3) can be generated by integrating out massive mediator states that couple directly to both the visible and dark sectors. Such mediators can give rise to new and unusual signals at high-energy colliders such as the Large Hadron Collider (LHC), either through their direct production or by providing a new avenue to populate the light states in the dark sector [14-16]. Discovering mediator particles or measuring their decoupling effects would also provide new insight into the structure and dynamics of the light states in the dark sector.

In this paper we investigate a very simple theory of mediators to a dark sector consisting of a $\mathrm{U}(1)_{x}$ vector boson $X$, first presented in ref. [17]. The mediators are an electroweak singlet $N$ and doublet $P$ of Dirac fermions with hypercharges $Y=0,-1 / 2$ and equal dark charge $q_{x}$. These quantum numbers allow for vector-like fermion masses and a coupling to the SM Higgs boson of the form

$$
-\mathscr{L} \supset \lambda \bar{P} \widetilde{H} N+\text { (h.c.) }
$$

where $\widetilde{H}=i \sigma_{2} H^{*}$. After electroweak symmetry breaking, the singlet and doublet mix to form a pair of neutral Dirac fermions $\psi_{1}$ and $\psi_{2}$, and a charged fermion $P^{-}$. We assume as well that the dark vector boson develops a mass $m_{x}$, either through a dark sector Higgs or Stueckelberg mechanism [18, 19]. The interaction of eq. (1.4) is analogous to the neutrino portal, but it involves the new $\mathrm{U}(1)_{x}$ charged mediators instead of the SM leptons; we call it the vectorized lepton portal. In addition to this portal interaction, loops of the new fermions also contribute to a vector portal coupling between the $\mathrm{U}(1)_{x}$ vector boson and hypercharge.

This general structure appears in a broad range of proposed extensions of the SM. The new fermions in the theory have the same SM quantum numbers as some of the models of vector-like leptons (without dark charges) considered in refs. [20, 21]. In theories with supersymmetry, superpotential couplings of the form of eq. (1.4) are the origin of general renormalizable Higgs portal interactions via scalar $F$-terms, and they have been 
invoked to connect the Higgs to gauge mediator supermultiplets [22, 23] and to increase the mass of the SM-like Higgs boson [24-26]. Closely related structures with non-Abelian dark gauge groups also emerge in some theories of neutral naturalness such as folded supersymmetry [27] and quirky little Higgs [28], and in relaxion constructions [29-32]. Realizations of the vectorized lepton portal with an Abelian dark group were studied in refs. [17, 33-35], and with a non-Abelian group in refs. [36, 37].

The vectorized lepton portal can induce a wide range of new experimental signals, both from the light dark vector and the heavier mediator fermions. The new signals of primary interest in this work are exotic decays of the SM Higgs boson. Loops of the vector-like fermions give rise to $h \rightarrow X X$ and $h \rightarrow X Z$ decay channels. We show that the resulting branching fractions can be much larger than from kinetic mixing alone. Furthermore, we also demonstrate that these decays are potentially observable at the LHC (and beyond) while being consistent with current bounds from precision electroweak tests and direct collider searches. Relative to the closely related previous works of refs. [17, 33], we compute the Higgs decay widths and the direct constraints due to the new fermions in more detail, and we show that current direct limits allow for observable Higgs signals at the LHC.

Following this introduction, we present a simple vectorized lepton portal model in more detail in section 2. Next, we calculate the Higgs boson decay widths to dark vectors through mediator fermion loops and discuss their observability at the LHC and beyond in section 3 . Constraints on the mediators from precision electroweak measurements, direct searches at the LHC, and stability of the Higgs potential are discussed in section 4. In section 5 we study the implications of the theory for dark matter and cosmology, and we discuss some potential extensions of the minimal theory motivated by them. Further extensions of the minimal theory to non-Abelian dark gauge groups are discussed in section 6. Finally, we reserve section 7 for our conclusions.

\section{Fields, masses, and interactions}

We consider a theory with two new vector-like fermion multiplets with charge assignments under $\mathrm{SU}(3)_{c} \times \mathrm{SU}(2)_{L} \times \mathrm{U}(1)_{Y} \times \mathrm{U}(1)_{x}$ of $N=\left(1,1,0 ; q_{x}\right)$ and $P=\left(1,2,-1 / 2 ; q_{x}\right)$. This allows the Yukawa coupling and masses:

$$
-\mathscr{L} \supset(\lambda \bar{P} \widetilde{H} N+\text { h.c. })+m_{P} \bar{P} P+m_{N} \bar{N} N,
$$

where $\widetilde{H}=i \sigma_{2} H^{*}$. Note that $m_{P}, m_{N}$, and $\lambda$ can all be taken to be real and positive through field redefinitions. We also normalize the dark gauge coupling $g_{x}$ such that either $q_{x}=1$ or $q_{x}=-1$. The set of fermion charges in our theory is minimal in that there is only one new (Dirac) field with SM gauge charges. Let us also mention that the Yukawa interaction of eq. (2.1) can be generalized to a chiral form with two independent Yukawa couplings that allows for $C P$ violation [38-41]; we focus on the parity-preserving form of eq. (2.1) for simplicity.

\subsection{Minimal masses and interactions}

Expanding the Higgs about its vacuum expectation value (VEV) in unitary gauge, $H \rightarrow$ $(v+h / \sqrt{2})$, with $v=174 \mathrm{GeV}$, and writing the $\mathrm{SU}(2)_{L}$ components of the doublet explicitly 
as $P=\left(P^{0}, P^{-}\right)^{T}$, the fermion terms become

$$
-\mathscr{L} \supset-m_{P} \bar{P}^{-} P^{-}+\left(\bar{N}, \bar{P}^{0}\right)\left(\begin{array}{cc}
m_{N} & \lambda v \\
\lambda v & m_{P}
\end{array}\right)\left(\begin{array}{c}
N \\
P^{0}
\end{array}\right)+\frac{\lambda}{\sqrt{2}} h\left(\bar{N} P^{0}+\bar{P}^{0} N\right) .
$$

The physical states are therefore a charged fermion $P^{-}$with mass $m_{P}$ together with two SM-neutral Dirac fermions $\psi_{1,2}$ with masses

$$
m_{1,2}=\frac{1}{2}\left[\left(m_{N}+m_{P}\right) \mp \sqrt{\left(m_{N}-m_{P}\right)^{2}+4 \lambda^{2} v^{2}}\right] .
$$

We only consider solutions with positive $m_{1}>0$ in this work, corresponding to the condition $\sqrt{m_{N} m_{P}}>\lambda v$, since the $m_{1}<0$ solution has $\left|m_{1}\right| \leq \lambda v$ and is strongly constrained by direct searches. The neutral gauge eigenstates are related to the mass eigenstates by

$$
\left(\begin{array}{c}
N \\
P^{0}
\end{array}\right)=\left(\begin{array}{cc}
c_{\alpha} & s_{\alpha} \\
-s_{\alpha} & c_{\alpha}
\end{array}\right)\left(\begin{array}{c}
\psi_{1} \\
\psi_{2}
\end{array}\right)
$$

with the mixing angle given by

$$
\tan (2 \alpha)=\frac{2 \lambda v}{m_{P}-m_{N}} .
$$

We choose the solution for $\alpha$ such that $m_{1}<m_{2}$.

Rewriting the Yukawa interaction in terms of the mass eigenstates, we find

$$
-\mathscr{L} \supset \frac{\lambda}{\sqrt{2}} h\left[2 s_{\alpha} c_{\alpha}\left(-\bar{\psi}_{1} \psi_{1}+\bar{\psi}_{2} \psi_{2}\right)+\left(c_{\alpha}^{2}-s_{\alpha}^{2}\right)\left(\bar{\psi}_{1} \psi_{2}+\bar{\psi}_{2} \psi_{1}\right)\right] .
$$

Note that the charged $P^{-}$state does not couple to the Higgs boson. The relevant vector boson couplings are

$$
\begin{aligned}
-\mathscr{L} \supset & \bar{g}\left(-\frac{1}{2}+s_{W}^{2}\right) Z_{\mu} \bar{P}^{-} \gamma^{\mu} P^{-}-e A_{\mu} \bar{P}^{-} \gamma^{\mu} P^{-} \\
& +\frac{g}{\sqrt{2}}\left[W_{\mu}^{+} \bar{P}^{-} \gamma^{\mu}\left(-s_{\alpha} \psi_{1}+c_{\alpha} \psi_{2}\right)+(\text { h.c. })\right] \\
& +\frac{1}{2} \bar{g} Z_{\mu}\left[s_{\alpha}^{2} \bar{\psi}_{1} \gamma^{\mu} \psi_{1}+c_{\alpha}^{2} \bar{\psi}_{2} \gamma^{\mu} \psi_{2}-s_{\alpha} c_{\alpha}\left(\bar{\psi}_{1} \gamma^{\mu} \psi_{2}+\bar{\psi}_{2} \gamma^{\mu} \psi_{1}\right)\right] \\
& +g_{x} X_{\mu}\left[\bar{\psi}_{1} \gamma_{\mu} \psi_{1}+\bar{\psi}_{2} \gamma_{\mu} \psi_{2}+\bar{P}^{-} \gamma_{\mu} P^{-}\right]
\end{aligned}
$$

where $\bar{g}=g / c_{W}=\sqrt{g^{2}+g^{\prime 2}}$.

Beyond the Yukawa and gauge couplings above, the dark sector also couples to the SM through gauge kinetic mixing $[10,11]$,

$$
-\mathscr{L} \supset \frac{\epsilon}{2 c_{W}} B_{\mu \nu} X^{\mu \nu}
$$

This interaction can be treated as in refs. [2, 3, 42], with the main effect for $m_{x} \ll m_{Z}$ being kinetic mixing with the photon with strength $\epsilon$. It allows the dark vector to decay to lighter SM final states. 
We take $\epsilon$ to be an independent parameter, but it should be noted that it is generated by $P$ loops. The log-enhanced running contribution to $\epsilon$ from these loops between scale $\mu$ and $m_{P}$ is $[43,44]$

$$
\begin{aligned}
\Delta \epsilon & \simeq-\frac{q_{x}}{3 \pi} \sqrt{\alpha_{x} \alpha} \ln \left(\frac{\mu}{m_{P}}\right) \\
& \simeq-q_{x}\left(3 \times 10^{-3}\right)\left(\frac{\alpha_{x}}{10 \alpha}\right)^{1 / 2} \ln \left(\frac{\mu}{m_{P}}\right),
\end{aligned}
$$

where $\alpha_{x}=g_{x}^{2} / 4 \pi$. Values much smaller than this are expected to require some degree of tuning, or additional structure in the theory such as an approximately conserved charge conjugation symmetry in the dark sector [34].

\subsection{Additional interactions}

Several other interactions can be added to the minimal set discussed above if the dark sector contains a scalar $\phi$ with dark charge $Q_{x}$, such as a dark Higgs boson responsible for generating the dark vector mass $[17,33]$. For any $Q_{x}$, the scalar can connect to the SM Higgs field through the Higgs portal,

$$
-\mathscr{L} \supset \kappa|\phi|^{2}|H|^{2}
$$

This will induce Higgs mixing if $\phi$ develops a VEV. Such an interaction is generated at two-loop order through the gauge and Yukawa couplings of the theory with size

$$
\begin{aligned}
\Delta \kappa & \sim \frac{Q_{x}^{2}}{(4 \pi)^{2}} \lambda^{2} \alpha_{x}^{2} \\
& =\left(4 \times 10^{-5}\right)\left(\frac{\alpha_{x}}{10 \alpha}\right)^{2} \lambda^{2} Q_{x}^{2} .
\end{aligned}
$$

As for $\epsilon$, we take this as a lower limit on the natural size of $\kappa$. It is parametrically smaller than the sizes of the effects we consider.

Other gauge invariant operators are possible for special values of the charge $Q_{x}$ of $\phi[17,33]$. For $Q_{x}= \pm q_{x}$, a direct lepton mixing is allowed,

$$
-\mathscr{L} \supset y_{L} \phi \bar{P}_{R} L_{L}+\text { (h.c.) }
$$

where $L_{L}$ is the SM lepton doublet. This operator can contribute to lepton masses and flavor violation, but current bounds can typically be satisfied for couplings below $\left|y_{L}\right| \lesssim$ $10^{-3}[17]$. With $Q_{x}=-2 q_{x}$ we can write

$$
-\mathscr{L} \supset y_{N} \phi \overline{N^{c}} N+\text { (h.c.) }
$$

which induces a Majorana mass for $N$ (and a one-loop contribution to $\kappa$ ) for non-zero $\langle\phi\rangle$. 


\section{Higgs boson decays to dark vectors}

Decays of the Higgs boson to one or more dark vectors are generated by the portal coupling of eq. (1.4). These arise at one-loop order from UV-finite triangle diagrams, in direct analogy to the contributions to the SM Higgs decay modes $h \rightarrow \gamma \gamma$ and $h \rightarrow g g$ from loops of the top quark. In our minimal vectorized lepton portal scenario, the new decay channels are $h \rightarrow X X$ and $h \rightarrow X Z$. We investigate these decays in this section.

Before proceeding, let us also mention that the mediator fermions typically do not modify the Higgs branching fractions to SM final states in a significant way. There is no direct one-loop contribution to $h \rightarrow g g$ since the mediators are uncolored, and the absence of a tree-level coupling of the charged $P^{-}$mode to the Higgs implies the same for $h \rightarrow \gamma \gamma$ and $h \rightarrow \gamma X$. The primary exception to this occurs when the new fermions are light enough that $h \rightarrow \psi_{1} \bar{\psi}_{1}$ is allowed. For $\lambda$ of order unity, this channel can easily dominate the Higgs width. Since our focus is on decays of the Higgs to dark vectors, which require larger $\lambda$ to be relevant, we concentrate on fermion masses greater than $m_{1}>m_{h} / 2$.

\subsection{Higgs branching fractions}

The vectorized lepton portal can induce both $h \rightarrow X X$ and $h \rightarrow X Z$ decays at a similar level. We collect in appendix A the loop functions relevant for the decay. The asymptotic form of the $h \rightarrow X X$ decay in the limit $m_{1,2} \gg m_{h}$ and $m_{x} \rightarrow 0$ can be obtained as a low-energy Higgs theorem [45]. The result is

$$
\begin{aligned}
\mathscr{L}_{\text {eff }} & \supset-\frac{1}{4} \frac{\alpha_{x}}{4 \pi}\left(\sum_{i=1}^{2} \Delta b_{i} \frac{2}{m_{i}} \frac{\partial m_{i}}{\partial v}\right)\left(\frac{h}{\sqrt{2}}\right) X_{\mu \nu} X^{\mu \nu} \\
& =-\frac{\alpha_{x}}{3 \pi} \frac{\lambda^{2} v}{m_{1} m_{2}}\left(\frac{h}{\sqrt{2}}\right) X_{\mu \nu} X^{\mu \nu},
\end{aligned}
$$

where $\Delta b_{i}=-4 / 3$, and corresponds to the gauge invariant effective operator

$$
\mathcal{L}_{\text {eff }} \supset-\frac{\alpha_{x}}{6 \pi} \frac{\lambda^{2}}{m_{1} m_{2}} H^{\dagger} H X_{\mu \nu} X^{\mu \nu} .
$$

We find the same result from the appropriate limit of the full loop calculation. ${ }^{1}$ The expression of eq. (3.3) shows that in the heavy fermion limit, the $h \rightarrow X X$ decay amplitude depends quadratically on the lepton portal Yukawa coupling $\lambda$ and the dark gauge coupling $g_{x}$, and decouples if either of the neutral modes becomes very heavy. Both features arise from the non-diagonal Higgs coupling to the $P$ and $N$ fields. A similar low-energy calculation can be performed for the $h \rightarrow X Z$ mode, but the result is less illuminating and does not correspond to a single gauge-invariant operator. However, the result scales approximately quadratically in $\lambda$ and linearly in $g_{x}$.

In figure 1 we show the range of Higgs branching fractions for $h \rightarrow X X$ (left) and $h \rightarrow X Z$ (right) in the $m_{P}-m_{N}$ plane due to fermion loops for $\lambda=1, \alpha_{x}=10 \alpha$, and $m_{x}=15 \mathrm{GeV}$. The solid (dashed) lines in the figure indicate contours of constant $\psi_{1}\left(\psi_{2}\right)$

\footnotetext{
${ }^{1}$ Our result is smaller by a factor of two than the related calculation of ref. [36].
} 

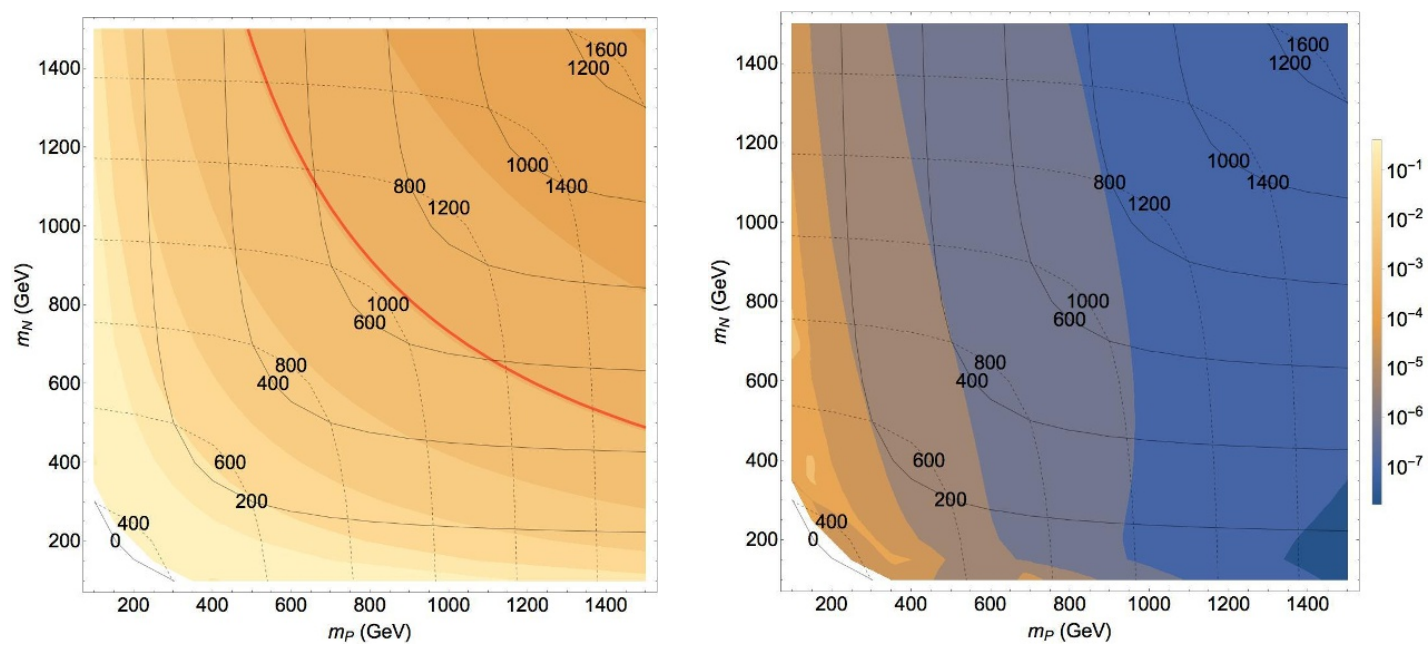

Figure 1. Branching ratios for $h \rightarrow X X$ (left) and $h \rightarrow X Z$ (right) decays due to mediator fermion loops in the $m_{P}-m_{N}$ plane for $\lambda=1, \alpha_{x}=10 \alpha$, and $m_{x}=15 \mathrm{GeV}$. The solid (dashed) black contours indicate $m_{1}\left(m_{2}\right)$ masses, while the solid red line shows the sensitivity of the most sensitive current LHC searches [46].

masses. For the $h \rightarrow X X$ channel, the branching fractions are symmetric in $m_{P}$ and $m_{N}$ since both states couple equally to the dark vector. The decay fractions for $h \rightarrow X Z$ tend to be somewhat lower than for $h \rightarrow X X$, due to weaker effective couplings in the amplitudes for large $\alpha_{x}$. In particular, the coupling of either fermion to the $Z$ is at most $\bar{g} / 2<\sqrt{10 \alpha_{x}}$ and is maximal for a doublet-like fermion, while the Higgs coupling relies on mixing between the $P^{0}$ and $N$ gauge eigenstates.

These exotic Higgs decay channels to one or more dark vectors also arise from the standard vector and Higgs portal couplings [47, 48]. With only a vector portal, the main new decay is $h \rightarrow X Z$ through the SM $h \rightarrow Z Z$ vertex with one of the $Z$ legs mixing into the dark vector $X[49,50]$. The corresponding decay width is suppressed by both $\epsilon^{2}$ and $\left(m_{x} / m_{Z}\right)^{2}$, and tends to have a very small branching fraction once other direct constraints on $\epsilon$ are taken into account [50]. The Higgs portal interaction can lead to $h \rightarrow X X$ decays with a significant rate $[47,48,50]$. We have not included the effects of these couplings in the results above. For minimal natural values of these parameters in our theory, we find that their contributions to the Higgs decay amplitudes are much smaller than those from direct fermion loops over the range of masses shown in the figure.

\subsection{Experimental signals}

Prospects for observing Higgs boson decays to one or more light dark vector bosons were studied in refs. [33, 47-55]. If the $X$ vector boson is the lightest state in the hidden sector, it decays exclusively to SM final states through its vector portal mixing with hypercharge. These decays can have a significant branching fraction to charged leptons [50], typically larger than that of the $Z$ boson, and are prompt for natural values of the kinetic mixing $\epsilon$.

The most recent experimental analysis of rare Higgs decays to dark vectors is the ATLAS study of ref. [46], based on about $20.5 \mathrm{fb}^{-1}$ of data at $\sqrt{s}=8 \mathrm{TeV}$. This search 
uses four-lepton final states with two opposite-sign same-flavor (OSSF) pairs, and covers the dark vector mass range $15 \mathrm{GeV} \leq m_{x} \leq m_{h} / 2$. For the $h \rightarrow X Z^{(*)}$ channel, the combined invariant mass is required to reconstruct the Higgs mass to within about $10 \mathrm{GeV}$, and a bump search is performed on the OSSF lepton pair with the lowest invariant mass. Their result can be translated into a limit on the branching ratio $\mathrm{BR}(h \rightarrow X Z) \lesssim 0.5-5 \times 10^{-3}$ over the dark vector mass range covered by the search. In the $h \rightarrow X X$ channel, events with two OSSF pairs are also selected and grouped such that the resulting pair of two-body invariant masses are as close as possible. The exclusion derived corresponds to $\mathrm{BR}(h \rightarrow$ $X X) \lesssim 3 \times 10^{-4}$ over the vector mass range studied.

Comparing these LHC exclusions to the branching fractions found above due to the mediator fermions of the vectorized lepton portal, figure 1 , we find that current data puts a significant limit on the new fermion masses for $\lambda=1$ and $\alpha_{x}=10 \alpha$. Dedicated analyses with the full current and expected LHC data sets will have sensitivity to even larger fermion masses in both the $h \rightarrow X X$ and $h \rightarrow X Z$ channels. Let us also point out that the search of ref. [46] concentrated on the dark vector mass range of $15 \mathrm{GeV} \leq m_{x} \leq m_{h} / 2$. This range is only weakly constrained by direct searches for dark vectors, with the strongest current bounds coming from precision electroweak tests that limit $\epsilon \lesssim 0.02$ [56]. The collider sensitivity to smaller dark vector masses is limited by backgrounds from heavy flavor resonances appearing at masses below about $11 \mathrm{GeV}$, and from the tendency of the leptons from a lighter vector boson to be collimated [57, 58]. Note, however, that existing direct limits on light dark vectors are much stronger for $m_{x} \lesssim 11 \mathrm{GeV}$ and constrain $\epsilon \lesssim 10^{-3}$ [5], of the same size as the natural range for this coupling in our minimal theory.

Our analysis shows that exotic Higgs decays to dark vectors from loops of heavy mediator fermions are potentially observable in future Higgs searches at the LHC and beyond. In the sections to follow, we investigate other constraints on the theory from precision electroweak tests, direct collider searches, Higgs stability, and dark matter considerations. In doing so, we set $\lambda=1, \alpha_{x}=10 \alpha$, and $m_{x}=15 \mathrm{GeV}$ as fiducial parameters against which to compare. For these parameters, we find that searches for exotic Higgs decay can provide comparable or greater sensitivity to the theory than other experimental probes.

\section{Precision electroweak and collider constraints}

The vectorized lepton portal can induce significant decay fractions for $h \rightarrow X X$ and $h \rightarrow$ $X Z$ provided $\lambda$ and $\alpha_{x}$ are relatively large and the vector-like fermions $\psi_{1}$ and $\psi_{2}$ are not too heavy. In this section we investigate the bounds imposed on the theory from precision electroweak measurements, direct collider searches, and Higgs stability.

\subsection{Electroweak constraints}

The new $\psi_{1}, \psi_{2}$, and $P^{-}$fermions couple directly to the electroweak vector bosons, and therefore induce oblique corrections to precision electroweak observables [59, 60]. In addition, the gauge kinetic mixing of $\mathrm{U}(1)_{x}$ with hypercharge leads to mixing between the physical $X, Z$, and $\gamma$ vector bosons, further modifying these observables [50, 56, 61-63]. However, for natural ranges of the kinetic mixing parameter $\epsilon \lesssim 10^{-2}$ with $m_{x} \lesssim 30 \mathrm{GeV}$, 


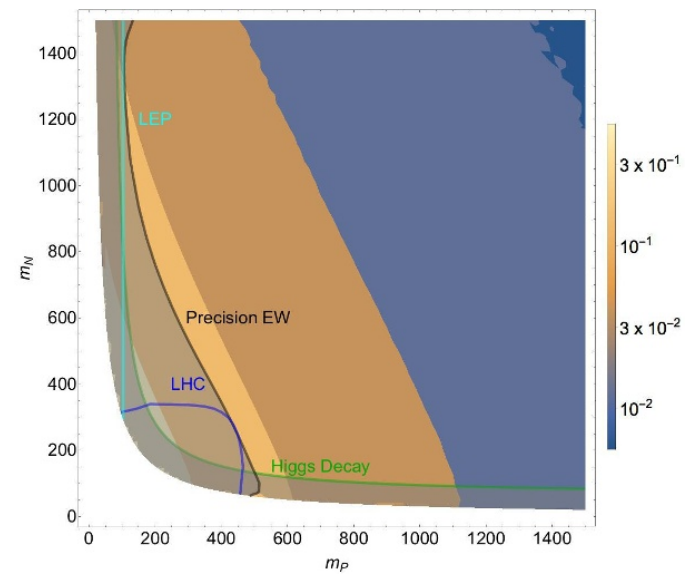

Figure 2. Precision electroweak and collider constraints on the minimal vectorized lepton portal for $\lambda=1$. The dark grey line shows the combined exclusion from precision electroweak tests, the cyan (LEP) and blue (LHC) indicate bounds from direct collider searches, and the green line shows the limit from the non-observation of invisible Higgs decays. The coloured contours indicate the shifts in the oblique $\Delta T$ parameter due to the heavy fermions.

the effects of vector boson mixing are much smaller than current limits $[50,56]$, and thus we focus exclusively on the effects of the heavy fermions.

Oblique corrections due to the new fermions are captured effectively by the PeskinTakeuchi $S, T$, and $U$ parameters. These have been computed for vector-like fermions with the same SM quantum numbers as those considered here in refs. [20,64]. Full expressions for the corrections to $S, T$, and $U$ are collected in appendix B.

To derive an exclusion on the theory from current electroweak data, we use the central values, uncertainties, and correlations among the $S, T$, and $U$ parameters obtained in the fit of ref. [65] with $m_{t}=173 \mathrm{GeV}$ and $m_{h}=125 \mathrm{GeV}$. The corresponding $95 \%$ c.l. excluded region in the $m_{P}-m_{N}$ plane for $\lambda=1$ lies to the left of the solid black line in figure 2 . We find that the corrections to $S$ and $U$ from the new fermions are typically very small, and the primary effect of the fermions is to shift the $T$ parameter, related to the mass splitting of the components of the electroweak doublet $P$ from mixing with $N$. Contours of $\Delta T$ are also shown in figure 2 , and the excluded region is approximated well by the condition $\Delta T \lesssim 0.14$

\subsection{Collider bounds}

Collider searches for the charginos and neutralinos of supersymmetry can be applied to the vector-like fermions we are considering. In particular, our system consists of an electroweak doublet and singlet, and is similar in its collider phenomenology to a Higgsino-Bino system [41]. The lightest new fermion in the theory is $\psi_{1}$, which is stable and contributes to missing energy in analogy to the lightest $\chi_{1}^{0}$ neutralino. We estimate here the limits on the $\psi_{1}, \psi_{2}$, and $P^{-}$massive fermions by reinterpreting searches for electroweak superpartners at LEP II and the LHC. 
For $m_{P} \ll m_{N}$, the lighter $\psi_{1}$ and $P^{-}$states both come mainly from the electroweak doublet and tend to be fairly close in mass, similar to a set of light Higgsinos $\left(\mu \ll M_{1}, M_{2}\right)$. In contrast to light Higgsinos, however, the neutral state is a single Dirac fermion $\psi_{1}$ instead of a pair of Marjorana $\chi_{1}$ and $\chi_{2}$ modes. The charged $P^{-}$state is similar to the lightest chargino $\chi_{1}^{+}$in its production, with decays through $P^{-} \rightarrow W^{-(*)} \psi_{1}$. The heaviest state $\psi_{2}$ is mostly singlet, and will therefore have suppressed production through electroweak vector bosons. With $m_{P} \ll m_{N}$, it decays in a roughly $2: 1: 1$ proportion via $\psi_{2} \rightarrow W^{+} P^{-}$, $\psi_{2} \rightarrow Z \psi_{1}$, and $\psi_{2} \rightarrow h \psi_{1}[66]$.

In the opposite limit, $m_{P} \gg m_{N}$, the lightest state $\psi_{1}$ is mostly singlet while the heavier $P^{-}$and $\psi_{2}$ particles are Higgsino-like. They decay via $P^{-} \rightarrow W^{-} \psi_{1}$, along with $\psi_{2} \rightarrow Z \psi_{1}$ and $\psi_{2} \rightarrow h \psi_{1}$ in a roughly $1: 1$ ratio [66]. This system is similar to the electroweakino sector of a supersymmetric theory with $M_{1} \ll \mu \ll M_{2}, m_{\text {sfermion }}$.

Searches for superpartners at LEP II are summarized in ref. [67]. The most relevant channels for our scenario are the chargino modes $e+e^{-} \rightarrow \chi_{1}^{+} \chi_{1}^{-}$with $\chi_{1}^{+} \rightarrow \chi_{1}^{0} W^{+(*)}$ [68]. These can be applied directly to $P^{+} P^{-}$production. For $\lambda=1$ and $m_{N} \lesssim 2 \mathrm{TeV}$, the chargino limits translate into

$$
m_{P}>103 \mathrm{GeV} .
$$

This value of $\lambda$ (and the condition $\sqrt{m_{N} m_{P}}>\lambda v$ mentioned in section 2) also implies that $\left(m_{1}+m_{2}\right)$ is always larger than the maximal LEP II center-of-mass energy, so no bounds are obtained from searches for $\chi_{1}^{0} \chi_{2}^{0}$ production in this case. Searches for neutralino LSPs with initial-state photon radiation can also be applied to $e^{+} e^{-} \rightarrow \psi_{1} \bar{\psi}_{1} \gamma$ [69], but we find production cross sections well below the limit from ref. [70].

More recently, the LHC collaborations have extended the constraints on electroweak superpartners to masses beyond the reach of LEP II. The new fermions in our theory can be produced by electroweak Drell-Yan channels, through an off-shell Higgs boson [71-73], and via an $s$-channel dark vector. Whenever the production cross section is large enough to be potentially observable, we find that it is dominated by standard Drell-Yan (for fiducial values of $\lambda=1$ and $\epsilon=10^{-3}$ ). Decays of the heavier fermions to the stable $\psi_{1}$ produce signals with jets, leptons, and missing energy in direct analogy to supersymmetric cascades. Radiation of dark vectors by these fermions can produce additional visible objects in the events [14-16]. We do not expect such radiation to have a significant qualitative effect on the searches considered here that rely mainly on leptons that reconstruct a $Z$ boson, but they could open new search channels at the LHC.

The most constraining LHC search for our theory appears to be the CMS opposite-sign same-flavor (OSSF) dilepton analysis of ref. [74]. This search was based on $35.9 \mathrm{fb}^{-1}$ of data at a center-of-mass energy of $\sqrt{s}=13 \mathrm{TeV}$. The channels in the analysis relevant for our theory were those designed for $\chi_{2}^{0} \chi_{1}^{ \pm}$production followed by $\chi_{2}^{0} \rightarrow \chi_{1}^{0} Z$ and $\chi_{1}^{ \pm} \rightarrow \chi_{1}^{0} W^{ \pm}$. These channels required exactly two isolated light-flavor OSSF leptons with $86 \mathrm{GeV}<$ $m_{\ell \ell}<96 \mathrm{GeV}$, at least two jets with $p_{T}>35 \mathrm{GeV}$ and $m_{j j}<110 \mathrm{GeV}$, and missing energy $\mathbb{E}_{T}>100 \mathrm{GeV}$. Vetoes on additional leptons and $b$-tagged jets were also applied. These channels will receive contributions from $\bar{\psi}_{2} P^{-}$and $\psi_{2} \bar{\psi}_{2}$ production with one onshell $\psi_{2} \rightarrow Z \psi_{1}$ decay. 
To estimate exclusion limits from this search, we use MadGraph5_aMC@NLO [75] with a model implemented in FeynRules [76] to compute the relevant LHC production cross sections at $\sqrt{s}=13 \mathrm{TeV}$. We then compare to the cross section limits obtained in ref. [74] for a Wino-like $\chi_{2}^{0} \chi_{1}^{ \pm}$simplified model in which the two electroweakino states are degenerate and assumed to decay exclusively to a stable $\chi_{1}^{0}$ state through the weak vector bosons. In making the comparison, we include $P^{-} \overline{\psi_{2}}, P^{+} \psi_{2}$, and $\psi_{2} \bar{\psi}_{2}$ production, and we rescale their cross sections by the branching fraction for $\psi_{2} \rightarrow Z \psi_{1}$. The main simplification we make in deriving our exclusions is the assumption that the detection efficiencies are approximately the same in our theory as for the simplified electroweakino model. We also take the exclusion cross section to be $\sigma_{\text {tot }}<0.01 \mathrm{pb}$. Both assumptions are somewhat agressive, and thus we expect our result to represent an upper limit on the exclusion derived from a full recasting of the CMS search. Our result is shown in figure 2.

Other potentially relevant LHC searches are the trilepton analysis of ref. [77] and the mass-degenerate dilepton analysis of ref. [78]. Comparing their excluded cross sections to those of our theory, we do not find any limits beyond the dilepton analysis described above.

\subsection{Higgs stability}

New fermions with large Yukawa couplings to the Higgs field can destabilize the Higgs potential. They do so by modifying the renormalization group (RG) evolution of the Higgs self coupling $\lambda_{H}$, and tend to drive it negative at a lower scale than in the SM [79-82]. Without additional new physics near the scale at which this occurs, the tunnelling rate from the standard electroweak vacuum to the unstable region at large Higgs field values tends to be shorter than the age of the universe [83, 84]. At best, this instability can be taken to be an upper cutoff for the consistency of the theory.

To investigate these effects, we evolve the couplings of the theory to higher scales using the one-loop RG equations for the system. These are listed in appendix C, and generalize the results of refs. [85-87]. As inputs, we use the $\overline{M S}$ values for the relevant SM parameters derived in ref. [88] defined at scale $\mu_{t}=173.34 \mathrm{GeV}$ :

$$
\begin{aligned}
g_{1} & =\sqrt{5 / 3}(0.3585), & g_{2} & =0.6476, \\
y_{t} & =0.9369, & \lambda_{H} & =0.12597 .
\end{aligned}
$$

These inputs are evolved up to the fiducial massive fermion scale $\mu_{F}=500 \mathrm{GeV}$ as in the $\mathrm{SM}$, and then from $\mu_{F}$ to higher scales in the full theory with heavy fermions and the dark vector boson.

As expected, we find that the new Yukawa coupling $\lambda$ drives the Higgs quartic coupling $\lambda_{H}$ negative more quickly than in the SM. The condition we apply for the metastability of the standard electroweak vacuum follows ref. [82], which is based on ref. [83],

$$
\lambda_{H}\left(\Lambda_{H}\right)=-0.065\left[1-0.02 \ln \left(\lambda_{H} / \mu_{t}\right)\right] .
$$

This relation defines $\Lambda_{H}$, the maximum scale at which new physics that stabilizes the Higgs potential must emerge. Numerically, $\Lambda_{H}$ tends to be one or two orders of magnitude larger than the scale at which the Higgs quartic coupling $\lambda_{H}$ runs negative [84]. 
For the inputs listed in eq. (4.2) together with $\lambda=1$ and $\alpha_{x}=10 \alpha$ at $\mu_{t}$, we find a Higgs instability cutoff scale of $\Lambda_{H} \simeq 4.6 \times 10^{4} \mathrm{GeV}$, with the quartic coupling running negative at $\mu \simeq 1.0 \times 10^{4} \mathrm{GeV}$. There is also a Landau pole in the new gauge coupling at scale $\mu \simeq 10^{11} \mathrm{GeV}$ for $\alpha_{x}\left(\mu_{t}\right)=10 \alpha$. Reducing $\alpha_{x}\left(\mu_{t}\right)$ quickly pushes up the scale at which the Landau pole occurs, but has only a mild (lowering) effect on $\Lambda_{H}$. The Higgs instability scale for $\lambda=1$ is relatively low, but is still high enough to justify our treatment of the heavy fermions provided we interpret the theory as an effective one with a cutoff near $5 \mathrm{TeV}$. Even so, we note that $\lambda=1$ is close to the upper limit of what is possible for the consistency of our previous analyses. ${ }^{2}$

\section{Connections to dark matter}

In the minimal realization of the vectorized lepton portal, the lightest exotic fermion $\psi_{1}$ is stable and contributes to the density of dark matter (DM). This state is also a Dirac fermion with direct couplings to the $Z^{0}$ and $X$ vector bosons, implying that it can have a large spin-independent scattering cross section with nuclei. We investigate these features in this section and show that they impose strong constraints on the model assuming standard thermal production of $\psi_{1}$ in the early universe. These constraints can be evaded in scenarios with low effective reheating temperatures or by going beyond the minimal realization of the theory.

\subsection{Relic densities}

The relic density of $\psi_{1}$ particles from thermal freeze-out is determined by its dominant annihilation cross sections to dark vectors, electroweak vectors, and Higgs final states. Annihilation to pairs of dark vector bosons in our scenario is identical to minimal models of secluded dark matter [8], with leading cross section

$$
\langle\sigma v\rangle_{X X} \simeq \frac{\pi \alpha_{x}^{2}}{m_{1}^{2}} \sqrt{1-\left(\frac{m_{x}}{m_{1}}\right)^{2}} .
$$

The complete expression can be found in ref. [89]. Since both the $N$ and $P$ states couple in the same way to the dark vector, this cross section is independent of their mixing, and depends only on the gauge coupling $\alpha_{x}$ and the mass $m_{1} \cdot^{3}$

For direct annihilation to SM final states, the most important modes are typically $\psi_{1} \overline{\psi_{1}} \rightarrow Z Z, W W, h h$. These cross sections depend sensitively on the mixing between the $P^{0}$ and $N$ gauge eigenstates that combine to make up $\psi_{1}$ and $\psi_{2}$. The $s$-wave amplitude for the $W W$ channel is facilitated by a $t$-channel $P^{-}$exchange and scales proportionally to $s_{\alpha}^{2}$, while the analogous $Z Z$ process involves $t$-channel $\psi_{1}$ or $\psi_{2}$ exchange and is proportional

\footnotetext{
${ }^{2}$ There is a significant sensitivity of these results to the SM input parameter values for small $\lambda \ll 1$ reflecting a theoretical uncertainty on our one-loop treatment. However, for $\lambda \sim 1$, the new Yukawa coupling dominates and the dependence on the SM inputs becomes modest.

${ }^{3} \mathrm{~A}$ light dark vector coupled to heavier dark matter can also enhance the annihilation cross section (in all channels) by the Sommerfeld effect [90-92]. We find that this enhancement is very mild for $\alpha_{x} \leq 10 \alpha$ and $m_{x}=15 \mathrm{GeV}$.
} 

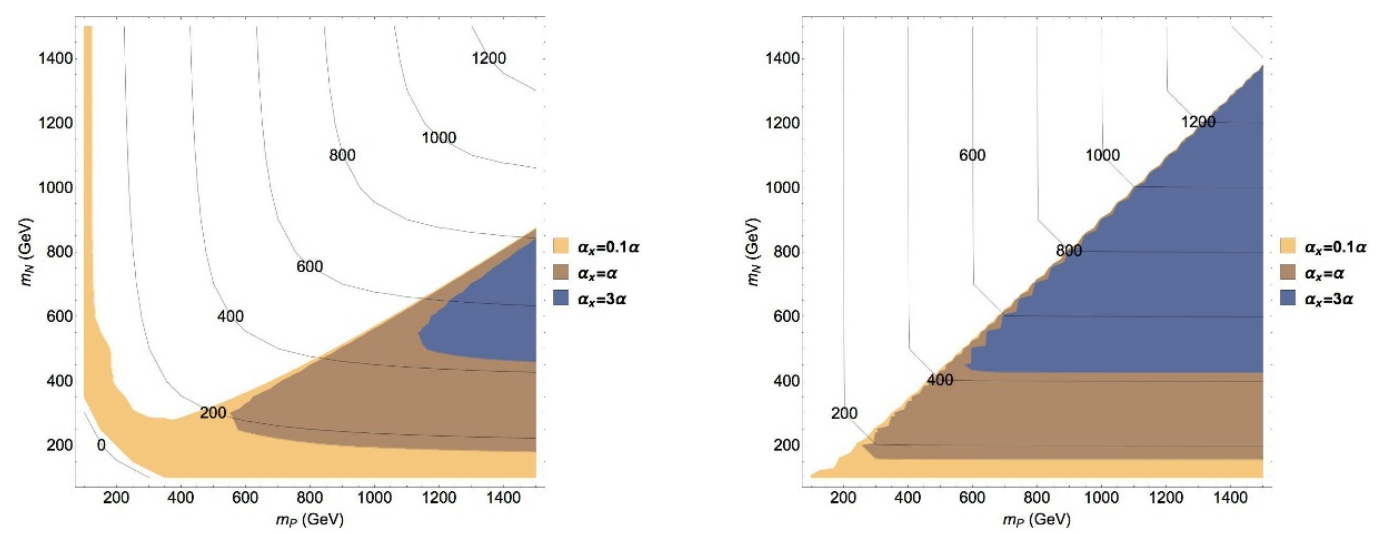

Figure 3. Regions in the $m_{P}-m_{N}$ where the thermal $\psi_{1}$ relic density exceeds the observed value. The plot on the left has $\lambda=1$, while the plot on the right corresponds to $\lambda=0.1$. The different shadings show the exclusions for $\alpha_{x}=0.1 \alpha, \alpha, 3 \alpha$. Also shown are contours of the $\psi_{1}$ mass $m_{1}$.

to $s_{\alpha}^{4}$ or $s_{\alpha}^{2} c_{\alpha}^{2}$ respectively. In the event of small mixing angles, $p$-wave processes involving an $s$-channel Higgs can be significant. These amplitudes scale as $\lambda s_{\alpha} c_{\alpha}$, and include $W W$, $Z Z$, or $h h$ final states.

In figure 3 we show regions in the $m_{P}-m_{N}$ plane where the $\psi_{1}$ relic density exceeds the observed value. In this figure, we set $\lambda=1$ (left) and $\lambda=0.1$ (right), with $m_{x}=11 \mathrm{GeV}$ and several values of $\alpha_{x}=0.1 \alpha, \alpha, 3 \alpha$. Setting $\alpha_{x}=10 \alpha$, the annihilation to dark vectors becomes very efficient and the entire parameter region shown yields an acceptable relic density. Also shown are contours of the $\psi_{1}$ mass $m_{1}$.

\subsection{Direct detection}

Direct searches for DM scattering put strong bounds on spin-independent DM-nucleon effective cross sections, on the order of $\sigma_{S I} \lesssim 10^{-46} \mathrm{~cm}^{2}$ for $m_{D M} \sim 100 \mathrm{GeV}$. This is orders or magnitude larger than the effective per-nucleon cross section of a stable Dirac fermion with electroweak charge, $\sigma_{S I} \simeq 10^{-39} \mathrm{~cm}^{-2}$ [93]. As a result, current direct detection bounds are sensitive to Dirac fermion relics that make up only a tiny fraction of the full dark matter density [94].

The spin-independent nucleon cross section of the $\psi_{1}$ state receives contributions from $Z, X$, and Higgs exchange. The corresponding effective operators have the same nonrelativistic limit and interfere with each other. Together, they imply an effective pernucleon cross section of [93]

$$
\sigma_{S I}=\frac{\mu_{n}^{2}}{\pi}\left[\frac{f_{p} Z+f_{n}(A-Z)}{A}\right]^{2}
$$

where $\mu_{n}$ is the DM-nucleon reduced mass, $A$ and $Z$ describe the target nucleus, and

$$
\begin{aligned}
& f_{p}=\frac{G_{F}}{\sqrt{2}} s_{\alpha}^{2}\left(1-4 s_{W}^{2}\right)-\frac{4 \pi}{m_{x}^{2}} \epsilon q_{x} \sqrt{\alpha \alpha_{x}}+\tilde{d}_{p}\left[\frac{2}{9}+\sum_{q} f_{T, q}^{p}\right], \\
& f_{n}=-\frac{G_{F}}{\sqrt{2}} s_{\alpha}^{2}+0+\tilde{d}_{n}\left[\frac{2}{9}+\sum_{q} f_{T, q}^{n}\right] .
\end{aligned}
$$


In both expressions above, the first term is due to $Z$ exchange, the second to $X$ exchange, and the third to Higgs exchange. The $X$ exchange terms depend on the sign of the dark charge of $\psi_{1}$ and assume $m_{x} \gtrsim 100 \mathrm{MeV}$. For the Higgs exchange terms, the quantity $\tilde{d}_{p, n}$ is given by

$$
\tilde{d}_{p, n}=\frac{m_{p, n}}{v} \frac{\lambda s_{\alpha} c_{\alpha}}{m_{h}^{2}},
$$

where the sums run over $q=u, d, s$, and the coefficients $f_{T, q}^{N}$ can be found in refs. [95-97].

Combining these expressions with the relic densities calculated previously, we find density-weighted per-nucleon cross sections, $\left(\Omega_{1} / \Omega_{D M}\right) \sigma_{S I}$, that are typically much larger than the current constraints from PandaX [98] and LUX [99]. This applies even for $\alpha_{x}=$ $10 \alpha$ when the $\psi_{1}$ relic density is significantly smaller than the total dark matter density. Dark photon exchange dominates for smaller $m_{x}$ and natural one-loop values of the kinetic mixing $\epsilon$. Even with this contribution suppressed by $\epsilon \rightarrow 0$, the scattering due to $Z$ exchange still tends to be too large.

Two potential loopholes to these bounds exist. The first requires a very small $\epsilon \rightarrow 0$ to suppress dark photon exchange together with a lighter singlet-like $\psi_{1}$ state to reduce the $Z$ and Higgs contributions to nucleon scattering. A large value of $\alpha_{x}$ is also needed to yield a small $\psi_{1}$ relic density. While these parameter values can give acceptably small density-weighted cross sections, they correspond to $s_{\alpha} \sim \lambda v / m_{P} \ll 1$ and imply a strong suppression of Higgs decays to dark photons. This is illustrated in the left panel of figure 4, where we show the parameter regions excluded by LUX [99] for $\lambda=0.1,0.3, \epsilon \rightarrow 0$, and $\alpha_{x}=10 \alpha$. The unshaded regions at the lower right are allowed.

The second loophole arises when there is a strong cancellation between the dark photon and $Z$ boson contributions to the cross section. Suppression of the cross section from such a cancellation is limited by the mixture of isotopes present in natural xenon to about $2 \times 10^{-4}$ relative to $f_{p}=f_{n}$. Moreover, the optimal suppression for xenon is different from that for other materials such as the germanium used in CDMS-II [100]. The allowed region of parameter space in this context for $\lambda=1, \epsilon=10^{-4}, m_{x}=10 \mathrm{GeV}$, and $\alpha_{x}=10 \alpha$ is illustrated in the right panel of figure 4 , where we show contours of the density weighted spin-independent cross section relative to the bound from LUX [99], $\left(\Omega_{1} / \Omega_{D M}\right) \sigma_{S I} / \sigma_{L U X}$. The region between the solid red lines is consistent with current limits.

\subsection{Beyond the minimal scenario}

Our analysis shows that the lightest $\psi_{1}$ fermion is very strongly constrained by dark matter direct detection, particularly when the Higgs branching fraction to dark vectors is significant. A similar conclusion was obtained in ref. [17]. These constraints can be avoided if there is non-thermal cosmological evolution or additional structure in the theory.

The relic densities used in making the estimates above assumed thermal cosmological evolution during and after the freezeout of the stable $\psi_{1}$ state. Much smaller relic densities can arise from non-thermal evolution. For example, late reheating following a period of inflation or matter domination with a reheating temperature below the freeze-out temperature of $\psi_{1}$ can yield a relic density that is orders of magnitude below the thermal 

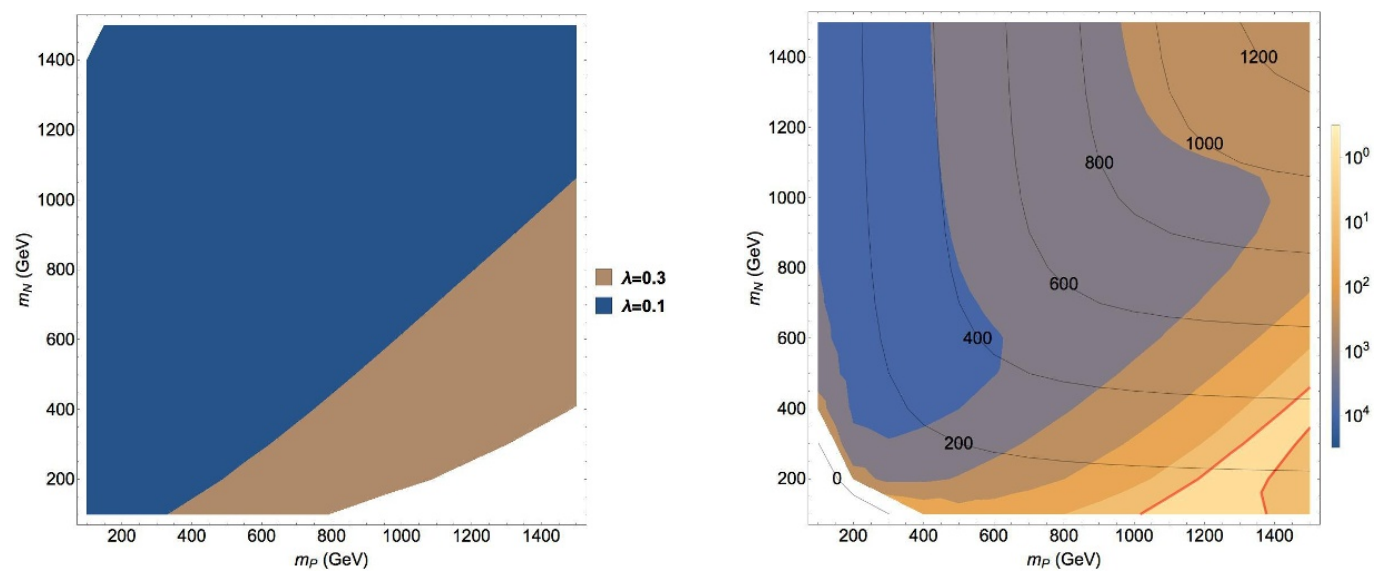

Figure 4. Two examples of how a thermal $\psi_{1}$ relic density with $\alpha_{x}=10 \alpha$ can be consistent with the current LUX bounds on spin-independent dark matter scattering [99]. The plot on the left illustrates the small $\lambda$ scenario, in which $\epsilon \rightarrow 0$, the lighter $\psi_{1}$ state is very singlet-like with suppressed mixing with the doublet. The unshaded regions at the lower right for $\lambda=0.1,0.3$ where the $\psi_{1}$ state is mostly singlet are allowed by current bounds. The right panel illustrates the scenario where the contributions to nucleon scattering from the dark photon and the $Z$ boson cancel against each other. This plot shows contours of $\left(\Omega_{1} / \Omega_{D M}\right) \sigma_{S I} / \sigma_{L U X}$ for $\lambda=1, \epsilon=10^{-4}$, and $m_{x}=10 \mathrm{GeV}$. The allowed region between the solid red lines.

value [101]. Even so, the tiny remaining abundance of $\psi_{1}$ could still be observable in direct detection experiments due to its large spin-dependent scattering cross section [94].

Constraints on the $\psi_{1}$ abundance from direct detection can also be reduced if it obtains a small Majorana mass or is able to decay [17]. A Majorana mass for $\psi_{1}$ can arise from the dark Higgs coupling listed in eq. (2.13). Such a mass term will split the four-component $\psi_{1}$ Dirac fermion into a pair of Majorana fermions, and thereby remove the dominant contribution to spin-independent elastic scattering from vector boson exchange [102]. The residual vector-mediated inelastic scattering is highly suppressed for mass splitting above about $\Delta m_{1}>200 \mathrm{keV}$. We find that the remaining spin-independent scattering due to Higgs excange can still be significant for $\lambda=1$, but it can lie below current limits for the subleading $\psi_{1}$ relic densities that occur for $\alpha_{x}=10 \alpha$. Alternatively, the operator of eq. (2.12) allows the $\psi_{1}$ state to decay to SM fermions through an electroweak vector boson, in which case the limits from dark matter searches are not relevant. While the coupling of eq. (2.12) is constrained by searches for lepton flavor violation, it is not difficult to avoid these limits while ensuring that $\psi_{1}$ decays occur before the onset of primordial nucleosynthesis.

\section{Comments on the non-Abelian case}

The vectorized lepton portal can also connect the SM to non-Abelian dark gauge groups. This arises in some theories addressing electroweak naturalness [27, 28], typically with a dark gauge group of $G_{x}=\mathrm{SU}(3)$, but the general structure can emerge more broadly [36, 103-106]. These more general groups can produce important changes in experimental 
observables compared to $G_{x}=\mathrm{U}(1)_{x}$. While many of these effects have been discussed in other contexts, we review them briefly here and point out a few particular features of our minimal construction. To be concrete, we focus here on $G_{x}=\mathrm{SU}\left(N_{x}\right)$ with $P$ and $N$ transforming in a complex representation $r$, as discussed in refs. [36, 104].

\subsection{Higgs decays to dark glueballs}

Instead of dark photons, the new vector bosons will be analagous to gluons. If there is no symmetry breaking in the dark sector and no other matter fields, the dark gluons will confine to form dark glueballs at the scale $\Lambda_{x}$. Here and for the rest of this section, we assume $\Lambda_{x} \ll m_{h}$ so that the direct effects of the new fermions can be treated in perturbation theory, and we define $\alpha_{x}$ to be the running dark coupling at scale $\mu=m_{h}$. The dark confinement scale is approximately

$$
\Lambda_{x} \simeq m_{h} \exp \left(-\frac{6 \pi}{11 N_{x} \alpha_{x}}\right) .
$$

This falls very quickly with decreasing $\alpha_{x}$ : for $N_{x}=3$ we find $\left(\alpha_{x}, \Lambda_{x}\right) \simeq(15 \alpha, 1 \mathrm{GeV})$, $(10 \alpha, 75 \mathrm{MeV}),(6.2 \alpha, 1 \mathrm{MeV})$, and $\left(\alpha, 10^{-27} \mathrm{MeV}\right)$.

The lightest dark glueball has quantum numbers $J^{P C}=0^{++}$and mass (for $N_{x}=3$ ) $m_{0} \simeq 6.8 \Lambda_{x}[107,108]$, but several other metastable glueballs arise as well. The effective Higgs interaction induced by the $N$ and $P$ fermions can be obtained by generalizing the calculation of section 3 :

$$
-\mathscr{L}_{\text {eff }} \supset \frac{\alpha_{x} T_{2}(r)}{6 \pi} \frac{\lambda^{2}}{m_{1} m_{2}} H^{\dagger} H X_{\mu \nu}^{a} X^{a \mu \nu} .
$$

After confinement and electroweak symmetry breaking, this operator induces a Higgs portal coupling between the $0^{++}$glueball and the SM Higgs boson, allowing it to decay with width [36]

$$
\Gamma_{0^{++}} \simeq\left[\frac{T_{2}(r)}{6 \pi} \frac{\lambda^{2}}{m_{1} m_{2}}\right]^{2}\left|\frac{\sqrt{2} v F_{S}}{m_{0}^{2}-m_{h}^{2}-i \Gamma_{h} m_{h}}\right|^{2} \Gamma_{h}\left(m_{0}\right),
$$

where $F_{S} \simeq\left(N_{x} / 3\right) 2.3 m_{0}^{3}$ is a glueball matrix element determined on the lattice [108, 109], and $\Gamma_{h}\left(m_{0}\right)$ is the decay width the SM Higgs would have if its mass were equal to $m_{0}$. Like the confinement scale, the glueball decay width varies extremely rapidly with the value of the running dark gauge coupling at $m_{h}$. Setting $\lambda=1$ and $\sqrt{m_{1} m_{2}}=500 \mathrm{GeV}$, we find a lifetime of $\tau \simeq 1 \mathrm{~s}$ for $\alpha_{x}\left(m_{h}\right)=12 \alpha$, and a decay length of $c \tau=1 \mathrm{~mm}$ for $\alpha_{x}\left(m_{h}\right)=23 \alpha{ }^{4}$

Higgs decays to dark glueballs also proceed through the operator of eq. (6.2) $[110,111]$. For light glueball masses, $m_{0} \ll m_{h} / 2$, the inclusive glueball branching fraction follows that for decays to dark photons up to a simple rescaling:

$$
\mathrm{BR}(h \rightarrow \text { glueballs }) \simeq \mathrm{BR}(h \rightarrow X X) \times \frac{T_{2}^{2}(r)\left(N_{x}^{2}-1\right)}{q_{x}^{4}} .
$$

\footnotetext{
${ }^{4}$ Fermion loops also yield dimension-eight operators connecting the dark gluons to SM vector bosons, but these yield much smaller decay widths for the parameter ranges of interest [103, 104].
} 
When $m_{0}$ approaches $m_{h} / 2$, resonances in the final state can modify the branching fraction in important ways $[110,111]$. Note as well that there is no $h \rightarrow(Z+$ glueballs $)$ decay channel in the absence of gauge symmetry breaking in the dark sector. The glueball final states from Higgs decays tend to be long-lived and appear as simple missing energy unless $\alpha_{x}\left(m_{h}\right)$ is much larger than $\alpha$. For moderate $\alpha_{x}\left(m_{h}\right)$, dedicated far detectors at the LHC could be sensitive to very late glueball decays [112]. Very large values of $\alpha_{x}\left(m_{h}\right)$ can give rise to displaced decays within ATLAS or CMS $[110,111]$, or produce emerging or semivisible jets $[113,114]$.

Other dark vector decay modes can arise when there is symmetry breaking in the dark sector above the confinement scale. For example, an adjoint dark Higgs field with a Yukawa coupling $\xi$ to the $N$ or $P$ fermions gives rise to the operator

$$
\mathcal{O} \sim \frac{\sqrt{\alpha_{x} \alpha} \xi T_{2}(r)}{4 \pi} \frac{1}{m} \Phi^{a} X_{\mu \nu}^{a} B^{\mu \nu},
$$

where $m_{\psi} \sim m_{1}, m_{2}$. This produces a kinetic mixing interaction for $\Phi^{a} \rightarrow\left\langle\Phi^{a}\right\rangle$, and could allow more rapid decays of (some of) the dark vector bosons $[9,115-117]$.

\subsection{Constraints}

Bounds from precision electroweak tests and Higgs stability are mostly independent of the low-energy dynamics of the dark sector. The shifts in the oblique parameters discussed in section 4.1 are enhanced by a factor of $d(r)$, the dimension of the $G_{x}$ representation of $N$ and $P$. For $G_{x}=\mathrm{SU}(3)$ with $r=\mathbf{3}$ and $\lambda=1$, this leads to an exclusion of $m_{P} \gtrsim 1000-400 \mathrm{GeV}$ for $m_{N}=0-1500 \mathrm{GeV}$.

The renormalization group equations relevant for a Higgs stability analysis with a general non-Abelian group $G_{x}$ and fermion representation $r$ are collected in appendix C. For a given value of $\lambda$, the bound from Higgs stability rapidly becomes more stringent as the dimension of the fermion representation increases. With $G_{x}=\mathrm{SU}(3), r=\mathbf{3}, \lambda=1$, and $\alpha_{x}=10 \alpha$, the Higgs stability cutoff approaches $\Lambda_{H} \simeq 3 \mathrm{TeV}$, only slightly above the range of explicit fermion masses we are considering. This situation can be improved somewhat by lowering the new Yukawa coupling modestly; reducing to $\lambda=0.8$ increases the stability cutoff scale to well over $10 \mathrm{TeV}$. The corresponding reduction in the Higgs branching fraction to dark vectors can be compensated by the color factors in eq. (6.4) and an increased dark gauge coupling. Note as well that in theories with non-Abelian dark (SU(3)) gauge groups motivated by electroweak naturalness, new physics is typically expected at scales below about $10 \mathrm{TeV}[27,28,110]$.

Direct collider searches for the massive fermions in the theory can be modified in more radical ways by an unbroken non-Abelian dark gauge group with $\Lambda_{x} \ll m_{1}, m_{2}$ [118-120]. Even so, we argue that our previous collider limits derived for the Abelian scenario can be applied here in many cases up to a rescaling by the fermion multiplicity $d(r)$. The first stages of fermion production and decay proceed much like in the Abelian case. Strong $G_{x}$ dynamics does not have a significant effect on fermion production (away from threshold), with the fermions created in pairs primarily by Drell-Yan processes. Next, the heavier $\psi_{2}$ and $P^{-}$states decay down to the lightest $\psi_{1}$ mode. For non-degenerate fermion masses, this typically occurs before the non-perturbative $G_{x}$ dynamics sets in. 
The immediate remnants of fermion production and electroweak cascade decays are therefore a $\psi_{1} \bar{\psi}_{1}$ pair. In contrast to the Abelian theory where they would leave the detector as missing energy, the fermions are now quirks and remain bound by a string of $G_{x}$ flux [118-120]. This string eventually pulls the fermions back together, causing them to oscillate until they annihilate [120]. Since the $\psi_{1}$ fermions do not carry colour or electromagnetic charge, they do not interact significantly with the material in collider detectors and they are not expected to be trapped. Their eventual annihilation products are dark glueballs, SM fermions, Higgs bosons, and $W$ and $Z$ vector bosons [120-125]. For $\Lambda_{x} \gtrsim 1 \mathrm{MeV}$, the dark glueball final states are the dominant decay products.

Our previous collider limits on the new fermions can be applied to the non-Abelian scenario as well when the dark glueballs are the dominant annihilation product and are long-lived. When these two conditions are met, the production modes and visible decay products are the nearly same as in the Abelian case up to an increased fermion multiplicity factor of $d(r)$. For $\Lambda_{x} \lesssim 1 \mathrm{MeV}$, visible annihilation final states of the $\psi_{1} \bar{\psi}_{1}$ pair would provide an additional search channel $[123,125]$. With larger $\Lambda_{x} \gtrsim 1 \mathrm{GeV}$, displaced decays of the dark glueballs could be visible [125].

\subsection{Dark matter considerations}

Thermal freezeout of $\psi_{1}$ proceeds similarly to the Abelian case, and can be treated in perturbation theory for $\Lambda_{x} \ll m_{1}$. If the annihilation is dominated by $\psi \bar{\psi}_{1} \rightarrow X X$ processes, the relic yield after freezeout is approximately

$$
m_{1} Y_{1}=m_{1}\left(\frac{n_{1}}{s}\right) \sim\left(10^{-11} \mathrm{GeV}\right)\left(\frac{m_{1}}{500 \mathrm{GeV}}\right)^{2}\left(\frac{10 \alpha}{\alpha_{x}}\right)^{2} .
$$

As the early universe cools to below $T \lesssim \Lambda_{x}$ after freezeout, the dark flux connections among the relic $\psi_{1}$ and $\bar{\psi}_{1}$ states become important. These induce a second stage of $\psi_{1} \bar{\psi}_{1}$ annihilation to dark glueballs and SM final states [120, 126, 127].

For $\Lambda_{x} \gtrsim 100 \mathrm{MeV}$, this secondary annhilation is expected to occur before the onset of primordial nucleosynthesis (BBN). However, smaller $\Lambda_{x}$ produces a later stage of secondary annihilation, and the annihilation products can disrupt light element abundances $[128,129]$ or the cosmic microwave background radiation [130,131]. We defer a full study of these effects to a future work, but we note that these considerations suggest that larger values of $\Lambda_{x} \gtrsim 100 \mathrm{MeV}$ are preferred if the new fermions and glueballs were ever thermalized in the early universe. ${ }^{5}$ Note that these constraints differ significantly from theories with quirks that carry QCD color, in which a second stage of QCD annihilation reduces the quirk relic densities to acceptable levels [120, 126, 127].

Cosmological constraints on relic fermions and glueballs can also be avoided if their pre-decay relic yield is significantly below the thermal estimate of eq. (6.6). This can occur in scenarios with low reheating temperatures [101], or even from the heavy $\psi_{1}$ fermions themselves if they come to dominate the energy density of the universe before they decay [134].

\footnotetext{
${ }^{5}$ Related considerations of relic glueball decays also tend to prefer larger $\Lambda_{x}$ values $[105,106,132,133]$.
} 


\section{Conclusions}

In this work we have studied the phenomenological consequences of the vectorized lepton portal, consisting of two or more new fermions that are charged under both the SM and a dark gauge force and that connect to the Higgs boson through a Yukawa coupling. The minimal realization consists of electroweak singlet and doublet fermions and an Abelian $\mathrm{U}(1)_{x}$ dark gauge group. These fermions act as mediators between the visible and dark sectors, and they induce a gauge kinetic mixing of the $\mathrm{U}(1)_{x}$ vector with hypercharge.

An important consequence of the mediator fermions is new exotic decay channels of the Higgs boson. In particular, fermion loops induce $h \rightarrow X X$ and $h \rightarrow X Z$ decays. The decay fractions of these modes are potentially observable at the LHC for larger values of the new Yukawa coupling $\lambda$ and the dark gauge coupling $\alpha_{x}$. We find that existing LHC searches for $h \rightarrow X X$ constrain the product of the new neutral fermion masses to be at least $\sqrt{m_{1} m_{2}} \gtrsim 850 \mathrm{GeV}$ for $\lambda=1, \alpha_{x}=10 \alpha$, and $m_{x}=15 \mathrm{GeV}$. This sensitivity to exotic Higgs decays can be significantly greater than direct limits on the new fermions from precision electroweak tests, collider searches, and Higgs stability considerations. Dark matter searches further constrain the new fermions, but the bounds depend on the evolution history of the cosmos. As a result, searches for exotic Higgs decays with future data from the LHC and beyond are a key discovery channel for scenarios of this type.

The minimal vectorized lepton portal studied here can also be extended in a number of ways. Expanding the new Yukawa coupling to a more general chiral form allows for $C P$ violation in Higgs decays to dark vectors $[17,135]$. If the dark sector has spontaneous symmetry breaking, the new fermions can potentially mix with SM leptons, leading to the violation of (charged) lepton flavor and introducing new interactios among neutrinos. The Abelian dark gauge group we have concentrated on can also be extended to non-Abelian groups with interesting consequences.

\section{Acknowledgments}

We thank Lindsay Forestell, David B. Kaplan, John Ng, Jessie Shelton, James Wells, and Richard Woloshyn for helpful discussions. This work is supported by the Natural Sciences and Engineering Research Council of Canada (NSERC), with DM supported in part by a Discovery Grant. TRIUMF receives federal funding via a contribution agreement with the National Research Council of Canada.

\section{A Higgs loop functions}

Higgs boson decays to $h \rightarrow X X$ and $h \rightarrow X Z$ are generated by loops of $\psi_{1}$ and $\psi_{2}$ fermions. All the relevant one-loop diagrams take the general form shown in figure 5 , which connects the SM Higgs to a pair of vectors $X$ and $Y$ with internal fermion states $a, b$, and $c$. It corresponds to a contribution to the amplitude of

$$
-i(\Delta \mathcal{M})=g_{a c h} g_{c b Y} g_{b a X} I^{\mu \nu} \varepsilon_{\mu}^{*}\left(k_{1}, \lambda_{1}\right) \varepsilon_{\nu}^{*}\left(k_{2}, \lambda_{2}\right)
$$




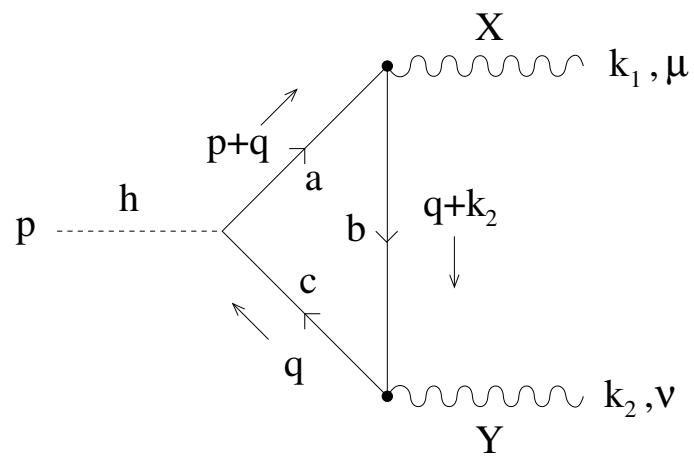

Figure 5. Loop diagram for $h \rightarrow X Y$ decay due to the fermion loop $\{a b c\}$.

with

$$
I^{\mu \nu}=-\int \frac{d^{d} q}{(2 \pi)^{d}} \frac{\operatorname{tr}\left[\left(\not+m_{c}\right) \gamma^{\nu}\left(\not{\alpha}+k_{1}+m_{b}\right) \gamma^{\mu}\left(\not+\not k+m_{a}\right)\right]}{\left.\left.\left(q^{2}-m_{c}^{2}+i \varepsilon\right)\left[\left(q+k_{1}-m_{b}\right)^{2}+i \varepsilon\right)\right]\left[\left(q+p-m_{a}\right)^{2}+i \varepsilon\right)\right]} .
$$

For each such diagram, there is a second independent diagram with the fermion arrows in figure 5 reversed of the form

$$
I I^{\mu \nu}=I^{\mu \nu}\left(a \leftrightarrow c, k_{1} \leftrightarrow k_{2}, \mu \leftrightarrow \nu\right) .
$$

Computing the diagram with dimensional regularization in $d=(4-\epsilon)$, we find

$$
\begin{array}{rl}
I^{\mu \nu}=\frac{4 i}{(4 \pi)^{2}} \int_{0}^{1} d x \int_{0}^{1-x} & d y\left(\frac{1}{\Delta}\left(m_{a} m_{b} m_{c} \eta^{\mu \nu}+m_{a} A^{\mu \nu}+m_{b} B^{\mu \nu}+m_{c} C^{\mu \nu}\right)\right. \\
\left.-\eta^{\mu \nu}\left[\left(m_{a}+m_{c}-m_{b}\right)+\left(2 m_{b}-m_{a}-m_{c}\right)\left(\frac{2}{\epsilon}+\ldots\right)\right]\right),
\end{array}
$$

where

$$
\begin{aligned}
A^{\mu \nu}= & {\left[-x^{2} m_{h}^{2}+\left(x+y-2 x y-y^{2}\right) m_{Y}^{2}+(x-2 x y)\left(k_{1} \cdot k_{2}\right)\right] \eta^{\mu \nu} } \\
& +\left(-x+2 x^{2}+2 x y\right) k_{1}^{\nu} k_{2}^{\mu} \\
B^{\mu \nu}= & {\left[\left(-x+x^{2}\right) m_{h}^{2}+\left(-y+2 x y+y^{2}\right) m_{Y}^{2}+(-y+2 x y)\left(k_{1} \cdot k_{2}\right)\right] \eta^{\mu \nu} } \\
& +y k_{1}^{\nu} k_{2}^{\mu} \\
C^{\mu \nu}= & {\left[\left(x-x^{2}\right) m_{h}^{2}+\left(-1+x+2 y-2 x y-y^{2}\right) m_{Y}^{2}\right.} \\
& \left.+(-1+x+y-2 x y)\left(k_{1} \cdot k_{2}\right)\right] \eta^{\mu \nu}+\left(1-3 x-y+2 x^{2}+2 x y\right) k_{1}^{\nu} k_{2}^{\mu}
\end{aligned}
$$

as well as

$$
\begin{aligned}
\Delta=\Delta_{a b c}= & x m_{a}^{2}+y m_{b}^{2}+z m_{c}^{2} \\
& +\left(-x+x^{2}\right) m_{h}^{2}+\left(-y+2 x y+y^{2}\right) m_{Y}^{2}+2 x y\left(k_{1} \cdot k_{2}\right) .
\end{aligned}
$$

The second loop $I I^{\mu \nu}$ can be obtained from this result by exchanging $a \leftrightarrow c$ everywhere. 
For $h \rightarrow X X$, the loops are $\{a b c\}=\{111\},\{222\}$. With $m_{a}=m_{b}=m_{c}$, we have $I^{\mu \nu}(a a a)=I I^{\mu \nu}(a a a)$ and the would-be divergent parts in eq. (A.4) cancel independently in each. The relevant coupling products are

$$
\begin{aligned}
& g_{11 h} g_{11 X} g_{11 X}=\left(-\sqrt{2} \lambda s_{\alpha} c_{\alpha}\right) g_{x}^{2} \\
& g_{22 h} g_{22 X} g_{22 X}=\left(+\sqrt{2} \lambda s_{\alpha} c_{\alpha}\right) g_{x}^{2} .
\end{aligned}
$$

Note the relative sign.

In the case of $h \rightarrow X Z$, the loops are $\{a b c\}=\{111\},\{222\},\{112\},\{221\}$, and the relevant coupling products are

$$
\begin{aligned}
& g_{11 h} g_{11 X} g_{11 Z}=\left(-\sqrt{2} \lambda s_{\alpha} c_{\alpha}\right)\left(g_{x} q_{x}\right)\left(\bar{g} s_{\alpha}^{2} / 2\right) \\
& g_{22 h} g_{22 X} g_{22 Z}=\left(+\sqrt{2} \lambda s_{\alpha} c_{\alpha}\right)\left(g_{x} q_{x}\right)\left(\bar{g} c_{\alpha}^{2} / 2\right) \\
& g_{21 h} g_{11 X} g_{12 Z}=\left[\lambda\left(c_{\alpha}^{2}-s_{\alpha}^{2}\right) / \sqrt{2}\right]\left(g_{x} q_{x}\right)\left(-\bar{g} s_{\alpha} c_{\alpha} / 2\right) \\
& g_{12 h} g_{22 X} g_{21 Z}=g_{21 h} g_{11 X} g_{12 Z},
\end{aligned}
$$

where $\bar{g}=\sqrt{g^{2}+g^{\prime 2}}$. For $\{111\}$ and $\{222\}$, the would-be divergent terms in eq. (A.4) cancel independently, while for $\{112\}$ and $\{221\}$ they cancel when the two contributions to the amplitude are summed.

\section{B Electroweak self-energies}

The relevant loop functions in $d=(4-\epsilon)$ dimensions are

$$
\begin{aligned}
4 \pi^{2} L_{a b}\left(p^{2}\right)= & {\left[\frac{1}{2}\left(m_{a}-m_{b}\right)^{2}-\frac{1}{3} p^{2}\right]\left[\frac{2}{\epsilon}-\gamma_{E}+\ln (4 \pi)-\ln \left(\frac{p^{2}}{\mu^{2}}\right)\right] } \\
& +\left[\left(m_{a} m_{b}-m_{a}^{2}\right) \widetilde{b}_{0}+\left(m_{a}^{2}-m_{b}^{2}+2 p^{2}\right) \widetilde{b}_{1}-2 p^{2} \widetilde{b}_{2}\right],
\end{aligned}
$$

where $\mu$ is the renormalization scale and

$$
\begin{aligned}
\widetilde{b}_{0}\left(p, m_{a}, m_{b}\right) & =\sum_{i= \pm}\left[\ln \left(1-x_{i}\right)-x_{i} \ln \left(1-\frac{1}{x_{i}}\right)-1\right] \\
2 \widetilde{b}_{1}\left(p, m_{a}, m_{b}\right) & =\sum_{i= \pm}\left[\ln \left(1-x_{i}\right)-x_{i}^{2} \ln \left(1-\frac{1}{x_{i}}\right)-x_{i}-\frac{1}{2}\right] \\
3 \widetilde{b}_{2}\left(p, m_{a}, m_{b}\right) & =\sum_{i= \pm}\left[\ln \left(1-x_{i}\right)-x_{i}^{3} \ln \left(1-\frac{1}{x_{i}}\right)-x_{i}^{2}-\frac{x_{i}}{2}-\frac{1}{3}\right]
\end{aligned}
$$

in which the index $i$ labels

$$
x_{ \pm}=\frac{1}{2 p^{2}}\left[\left(p^{2}+m_{a}^{2}-m_{b}^{2}\right) \pm \sqrt{\left(p^{2}+m_{a}^{2}-m_{b}^{2}\right)^{2}-4 p^{2}\left(m_{a}^{2}-i \varepsilon\right)}\right],
$$

and the $i \varepsilon$ defines the proper branch of the logarithms when their arguments become negative or complex. These loop functions are closely related to (the finite parts) of the 
Passarino-Veltman functions [136]. For $p^{2} \rightarrow 0$, the result simplifies to

$$
\begin{aligned}
4 \pi^{2} L_{a b}\left(p^{2}\right)= & \frac{1}{2}\left(m_{a}-m_{b}\right)^{2}\left[\frac{2}{\epsilon}-\gamma_{E}+\ln (4 \pi)-\ln \left(\frac{m_{a} m_{b}}{\mu^{2}}\right)+\frac{1}{2}\right] \\
& -\frac{1}{2} m_{a} m_{b}-\frac{1}{4\left(m_{a}^{2}-m_{b}^{2}\right)} \ln \left(\frac{m_{a}^{2}}{m_{b}^{2}}\right)\left(m_{a}^{4}-2 m_{a}^{3} m_{b}-2 m_{a} m_{b}^{3}+m_{b}^{4}\right) .
\end{aligned}
$$

In terms of these loop functions, the shifts in the oblique parameters $S, T$, and $U$ due to the vector-like fermions are $[59,60]$

$$
\begin{gathered}
\Delta S=\frac{4 \pi}{m_{Z}^{2}}\left(-\left[L_{--}\left(m_{Z}^{2}\right)-L_{--}(0)\right]\right. \\
\left.+s_{\alpha}^{4}\left[L_{11}\left(m_{Z}^{2}\right)-L_{11}(0)\right]+2 c_{\alpha}^{2} s_{\alpha}^{2}[12]+c_{\alpha}^{4}[22]\right) \\
\Delta S+\Delta U=\frac{8 \pi}{c_{W}^{2} m_{Z}^{2}}\left(s_{\alpha}^{2}\left[L_{1-}\left(m_{W}^{2}\right)-L_{1-}(0)\right]+c_{\alpha}^{2}\left[L_{2-}\left(m_{W}^{2}\right)-L_{2-}(0)\right]\right. \\
\left.\quad-c_{W}^{2}\left[L_{--}\left(m_{Z}^{2}\right)-L_{--}(0)\right]\right) \\
\Delta T=\frac{2 \pi}{s_{W}^{2} c_{W}^{2} m_{Z}^{2}}\left[s_{\alpha}^{2} L_{1-}(0)+c_{\alpha}^{2} L_{2-}(0)-s_{\alpha}^{2} c_{\alpha}^{2} L_{12}(0)\right] .
\end{gathered}
$$

These expressions are independent of $1 / \epsilon$ and the renormalization scale $\mu$.

\section{Renormalization group equations}

We collect here the one-loop renormalization group (RG) equations relevant for the Higgs stability analysis of section 4.3. In these equations, the only the SM Yukawa coupling we keep is that of the top quark, and we use the SU(5) normalization for the hypercharge coupling, $g_{1}=\sqrt{5 / 3} g^{\prime}$. Our normalization for the Higgs self coupling is $V(H) \supset \lambda_{H}|H|^{4}$ so that $\lambda_{H} \simeq m_{h}^{2} / 2 v^{2}$ with $v \simeq 174 \mathrm{GeV}$. To allow for generalization beyond the minimal Abelian vectorized lepton portal theory, we write the RG equations for general dark gauge group $G_{x}$ under which $P$ and $N$ transform under the representation $r_{x}$ with dimension $d\left(r_{x}\right)$.

With these assumptions, the RG equations for the system above the heavy fermion threshold can be adapted from the general results of refs. [85-87] as in refs. [79-82]. We find

$$
\begin{aligned}
(4 \pi)^{2} \frac{d \lambda_{H}}{d t}= & 24 \lambda_{H}^{2}+4 \lambda_{H}\left[3 y_{t}^{2}+2 d\left(r_{x}\right) \lambda^{2}\right]-2\left[3 y_{t}^{4}+2 d\left(r_{x}\right) \lambda^{4}\right] \\
& -3 \lambda_{H}\left(3 g_{2}^{2}+\frac{3}{5} g_{1}^{2}\right)+\frac{3}{8}\left[2 g_{2}^{4}+\left(g_{2}^{2}+\frac{3}{5} g_{1}^{2}\right)^{2}\right] \\
(4 \pi)^{2} \frac{d y_{t}}{d t}= & \frac{9}{2} y_{t}^{3}+2 d\left(r_{x}\right) y_{t} \lambda^{2}-y_{t}\left(8 g_{3}^{2}+\frac{9}{4} g_{2}^{2}+\frac{17}{20} g_{1}^{2}\right) \\
(4 \pi)^{2} \frac{d \lambda}{d t}= & {\left[\frac{3+4 d\left(r_{x}\right)}{2}\right] \lambda^{3}+3 \lambda y_{t}^{2}-\lambda\left[\frac{9}{4} g_{2}^{2}+\frac{9}{20} g_{1}^{2}+6 C_{2}\left(r_{x}\right) g_{x}^{2}\right] }
\end{aligned}
$$


together with

$$
\begin{aligned}
(4 \pi)^{2} \frac{d g_{2}}{d t} & =\left[-\frac{19}{6}+\frac{2}{3} d\left(r_{x}\right)\right] g_{2}^{3} \\
(4 \pi)^{2} \frac{d g_{1}}{d t} & =\left[\frac{41}{10}+\frac{2}{5} d\left(r_{x}\right)\right] g_{1}^{3} \\
(4 \pi)^{2} \frac{d g_{x}}{d t} & =\left[-\frac{11}{3} C_{2}\left(G_{x}\right)+4 S_{2}\left(r_{x}\right)\right] g_{x}^{3},
\end{aligned}
$$

where $t=\ln \left(\mu / \mu_{0}\right)$ defines the renormalization scale, and $S_{2}\left(r_{x}\right)$ and $C_{2}\left(r_{x}\right)$ refer to the trace and Casimir invariants of the representation $r_{x}$ of $N$ and $P$ under $G_{x}$.

For $G_{x}=\mathrm{U}(1)_{x}$ with $N_{f}$ copies of the $N$ and $P$ fields of charge $q_{x}$, we have

$$
C_{2}\left(r_{x}\right)=q_{x}^{2}, \quad S_{2}\left(r_{x}\right)=q_{x}^{2} N_{f}, \quad d\left(r_{x}\right)=N_{f}, \quad C_{2}\left(G_{x}\right)=0 .
$$

This case also allows for kinetic mixing between hypercharge and $\mathrm{U}(1)_{x}$. The corresponding evolution equation for the mixing parameter $\tilde{\epsilon}=\epsilon / c_{W}$ above the heavy fermion mass threshold is $[43,44]$

$$
(4 \pi)^{2} \frac{d \tilde{\epsilon}}{d t}=4 N_{f} \tilde{\epsilon}\left(g_{x} q_{x}\right)^{2}+\left(\frac{41}{10}+\frac{2}{5} N_{f}\right) \tilde{\epsilon} g_{1}^{2}-\frac{8}{3} \sqrt{\frac{3}{5}} N_{f} g_{1}\left(g_{x} q_{x}\right) .
$$

Below the heavy fermion masses, the remaining evolution is homogeneous in $\tilde{\epsilon}$. There is also a small correction to the running of $g_{1}$ and $g_{x}$ proportional to $\tilde{\epsilon}$ that we do not include.

Open Access. This article is distributed under the terms of the Creative Commons Attribution License (CC-BY 4.0), which permits any use, distribution and reproduction in any medium, provided the original author(s) and source are credited.

\section{References}

[1] P. Fayet, U-boson production in $e^{+} e^{-}$annihilations, $\psi$ and $\Upsilon$ decays and light dark matter, Phys. Rev. D 75 (2007) 115017 [hep-ph/0702176] [INSPIRE].

[2] M. Pospelov, Secluded U(1) below the weak scale, Phys. Rev. D 80 (2009) 095002 [arXiv: 0811.1030] [INSPIRE].

[3] J.D. Bjorken, R. Essig, P. Schuster and N. Toro, New fixed-target experiments to search for dark gauge forces, Phys. Rev. D 80 (2009) 075018 [arXiv:0906.0580] [INSPIRE].

[4] R. Essig et al., Working group report: new light weakly coupled particles, arXiv:1311.0029 [INSPIRE].

[5] J. Alexander et al., Dark sectors 2016 workshop: community report, arXiv:1608.08632 [INSPIRE].

[6] C. Boehm and P. Fayet, Scalar dark matter candidates, Nucl. Phys. B 683 (2004) 219 [hep-ph/0305261] [INSPIRE].

[7] N. Borodatchenkova, D. Choudhury and M. Drees, Probing MeV dark matter at low-energy $e^{+} e^{-}$colliders, Phys. Rev. Lett. 96 (2006) 141802 [hep-ph/0510147] [InSPIRE]. 
[8] M. Pospelov, A. Ritz and M.B. Voloshin, Secluded WIMP dark matter, Phys. Lett. B 662 (2008) 53 [arXiv:0711.4866] [INSPIRE].

[9] N. Arkani-Hamed, D.P. Finkbeiner, T.R. Slatyer and N. Weiner, A theory of dark matter, Phys. Rev. D 79 (2009) 015014 [arXiv:0810.0713] [InSPIRE].

[10] L.B. Okun, Limits of electrodynamics: paraphotons?, Sov. Phys. JETP 56 (1982) 502 [Zh. Eksp. Teor. Fiz. 83 (1982) 892] [INSPIRE].

[11] B. Holdom, Two U(1)'s and $\epsilon$ charge shifts, Phys. Lett. B 166 (1986) 196 [INSPIRE].

[12] R.M. Schabinger and J.D. Wells, A Minimal spontaneously broken hidden sector and its impact on Higgs boson physics at the large hadron collider, Phys. Rev. D 72 (2005) 093007 [hep-ph/0509209] [INSPIRE].

[13] B. Patt and F. Wilczek, Higgs-field portal into hidden sectors, hep-ph/0605188 [INSPIRE].

[14] M.J. Strassler and K.M. Zurek, Echoes of a hidden valley at hadron colliders, Phys. Lett. B 651 (2007) 374 [hep-ph/0604261] [INSPIRE].

[15] M.J. Strassler, Possible effects of a hidden valley on supersymmetric phenomenology, hep-ph/0607160 [INSPIRE].

[16] T. Han, Z. Si, K.M. Zurek and M.J. Strassler, Phenomenology of hidden valleys at hadron colliders, JHEP 07 (2008) 008 [arXiv:0712.2041] [INSPIRE].

[17] H. Davoudiasl, H.-S. Lee and W.J. Marciano, Dark side of Higgs diphoton decays and muon g-2, Phys. Rev. D 86 (2012) 095009 [arXiv:1208.2973] [inSPIRE].

[18] E.C.G. Stueckelberg, Interaction energy in electrodynamics and in the field theory of nuclear forces, Helv. Phys. Acta 11 (1938) 225 [InSPIRE].

[19] B. Körs and P. Nath, A Stueckelberg extension of the standard model, Phys. Lett. B 586 (2004) 366 [hep-ph/0402047] [INSPIRE].

[20] S.A.R. Ellis, R.M. Godbole, S. Gopalakrishna and J.D. Wells, Survey of vector-like fermion extensions of the Standard Model and their phenomenological implications, JHEP 09 (2014) 130 [arXiv:1404.4398] [INSPIRE].

[21] S. Bhattacharya, N. Sahoo and N. Sahu, Minimal vectorlike leptonic dark matter and signatures at the LHC, Phys. Rev. D 93 (2016) 115040 [arXiv:1510.02760] [INSPIRE].

[22] G.R. Dvali, G.F. Giudice and A. Pomarol, The Mu problem in theories with gauge mediated supersymmetry breaking, Nucl. Phys. B 478 (1996) 31 [hep-ph/9603238] [INSPIRE].

[23] N. Craig, S. Knapen, D. Shih and Y. Zhao, A complete model of low-scale gauge mediation, JHEP 03 (2013) 154 [arXiv: 1206.4086] [inSPIRE].

[24] A. Azatov, J. Galloway and M.A. Luty, Superconformal technicolor, Phys. Rev. Lett. 108 (2012) 041802 [arXiv: 1106.3346] [INSPIRE].

[25] J.J. Heckman, P. Kumar, C. Vafa and B. Wecht, Electroweak symmetry breaking in the DSSM, JHEP 01 (2012) 156 [arXiv:1108.3849] [INSPIRE].

[26] J.L. Evans, M. Ibe and T.T. Yanagida, The lightest Higgs boson mass in the MSSM with strongly interacting spectators, Phys. Rev. D 86 (2012) 015017 [arXiv:1204.6085] [INSPIRE].

[27] G. Burdman, Z. Chacko, H.-S. Goh and R. Harnik, Folded supersymmetry and the LEP paradox, JHEP 02 (2007) 009 [hep-ph/0609152] [INSPIRE]. 
[28] H. Cai, H.-C. Cheng and J. Terning, A quirky little Higgs model, JHEP 05 (2009) 045 [arXiv:0812.0843] [INSPIRE].

[29] P.W. Graham, D.E. Kaplan and S. Rajendran, Cosmological relaxation of the electroweak scale, Phys. Rev. Lett. 115 (2015) 221801 [arXiv:1504.07551] [INSPIRE].

[30] O. Antipin and M. Redi, The half-composite two Higgs doublet model and the relaxion, JHEP 12 (2015) 031 [arXiv: 1508.01112] [INSPIRE].

[31] B. Batell, G.F. Giudice and M. McCullough, Natural heavy supersymmetry, JHEP 12 (2015) 162 [arXiv: 1509.00834] [INSPIRE].

[32] K. Choi and S.H. Im, Realizing the relaxion from multiple axions and its UV completion with high scale supersymmetry, JHEP 01 (2016) 149 [arXiv:1511.00132] [INSPIRE].

[33] H. Davoudiasl, H.-S. Lee, I. Lewis and W.J. Marciano, Higgs decays as a window into the dark sector, Phys. Rev. D 88 (2013) 015022 [arXiv:1304.4935] [INSPIRE].

[34] A. DiFranzo, P.J. Fox and T.M.P. Tait, Vector dark matter through a radiative Higgs portal, JHEP 04 (2016) 135 [arXiv: 1512.06853] [INSPIRE].

[35] A. DiFranzo and G. Mohlabeng, Multi-component dark matter through a radiative Higgs portal, JHEP 01 (2017) 080 [arXiv:1610.07606] [INSPIRE].

[36] J.E. Juknevich, Pure-glue hidden valleys through the Higgs portal, JHEP 08 (2010) 121 [arXiv:0911.5616] [INSPIRE].

[37] H. Beauchesne, E. Bertuzzo and G. Grilli di Cortona, Constraints on the relaxion mechanism with strongly interacting vector-fermions, arXiv:1705.06325 [INSPIRE].

[38] R. Mahbubani and L. Senatore, The minimal model for dark matter and unification, Phys. Rev. D 73 (2006) 043510 [hep-ph/0510064] [INSPIRE].

[39] F. D'Eramo, Dark matter and Higgs boson physics, Phys. Rev. D 76 (2007) 083522 [arXiv:0705.4493] [INSPIRE].

[40] R. Essig, Direct detection of non-chiral dark matter, Phys. Rev. D 78 (2008) 015004 [arXiv: 0710.1668] [INSPIRE].

[41] T. Cohen, J. Kearney, A. Pierce and D. Tucker-Smith, Singlet-doublet dark matter, Phys. Rev. D 85 (2012) 075003 [arXiv: 1109.2604] [INSPIRE].

[42] H. Davoudiasl, H.-S. Lee and W.J. Marciano, 'Dark' Z implications for parity violation, rare meson decays and Higgs physics, Phys. Rev. D 85 (2012) 115019 [arXiv:1203.2947] [INSPIRE].

[43] F. del Aguila, G.D. Coughlan and M. Quirós, Gauge coupling renormalization with several U(1) factors, Nucl. Phys. B 307 (1988) 633 [Erratum ibid. B 312 (1989) 751] [InSPIRE].

[44] K.R. Dienes, C.F. Kolda and J. March-Russell, Kinetic mixing and the supersymmetric gauge hierarchy, Nucl. Phys. B 492 (1997) 104 [hep-ph/9610479] [INSPIRE].

[45] M.A. Shifman, A.I. Vainshtein, M.B. Voloshin and V.I. Zakharov, Low-energy theorems for Higgs boson couplings to photons, Sov. J. Nucl. Phys. 30 (1979) 711 [Yad. Fiz. 30 (1979) 1368] [INSPIRE].

[46] ATLAS collaboration, Search for new light gauge bosons in Higgs boson decays to four-lepton final states in pp collisions at $\sqrt{s}=8 \mathrm{TeV}$ with the ATLAS detector at the LHC, Phys. Rev. D 92 (2015) 092001 [arXiv:1505.07645] [INSPIRE]. 
[47] S. Gopalakrishna, S. Jung and J.D. Wells, Higgs boson decays to four fermions through an abelian hidden sector, Phys. Rev. D 78 (2008) 055002 [arXiv:0801.3456] [INSPIRE].

[48] D. Curtin et al., Exotic decays of the 125 GeV Higgs boson, Phys. Rev. D 90 (2014) 075004 [arXiv: 1312.4992] [INSPIRE].

[49] A. Falkowski and R. Vega-Morales, Exotic Higgs decays in the golden channel, JHEP 12 (2014) 037 [arXiv: 1405.1095] [InSPIRE].

[50] D. Curtin, R. Essig, S. Gori and J. Shelton, Illuminating dark photons with high-energy colliders, JHEP 02 (2015) 157 [arXiv:1412.0018] [INSPIRE].

[51] E. Gabrielli, M. Heikinheimo, B. Mele and M. Raidal, Dark photons and resonant monophoton signatures in Higgs boson decays at the LHC, Phys. Rev. D 90 (2014) 055032 [arXiv:1405.5196] [INSPIRE].

[52] S. Biswas, E. Gabrielli, M. Heikinheimo and B. Mele, Higgs-boson production in association with a dark photon in $e^{+} e^{-}$collisions, JHEP 06 (2015) 102 [arXiv: 1503.05836] [INSPIRE].

[53] S. Biswas, E. Gabrielli, M. Heikinheimo and B. Mele, Dark-photon searches via Higgs-boson production at the LHC, Phys. Rev. D 93 (2016) 093011 [arXiv:1603.01377] [INSPIRE].

[54] S. Biswas, E. Gabrielli, M. Heikinheimo and B. Mele, Dark-photon searches via ZH production at $e^{+} e^{-}$colliders, arXiv:1703.00402 [INSPIRE].

[55] M.D. Campos, D. Cogollo, M. Lindner, T. Melo, F.S. Queiroz and W. Rodejohann, Neutrino masses and absence of flavor changing interactions in the 2HDM from gauge principles, arXiv: 1705.05388 [INSPIRE].

[56] A. Hook, E. Izaguirre and J.G. Wacker, Model independent bounds on kinetic mixing, Adv. High Energy Phys. 2011 (2011) 859762 [arXiv: 1006. 0973] [INSPIRE].

[57] A. Falkowski, J.T. Ruderman, T. Volansky and J. Zupan, Hidden Higgs decaying to lepton jets, JHEP 05 (2010) 077 [arXiv: 1002.2952] [INSPIRE].

[58] A. Falkowski, J.T. Ruderman, T. Volansky and J. Zupan, Discovering Higgs decays to lepton jets at hadron colliders, Phys. Rev. Lett. 105 (2010) 241801 [arXiv:1007.3496] [INSPIRE].

[59] M.E. Peskin and T. Takeuchi, A new constraint on a strongly interacting Higgs sector, Phys. Rev. Lett. 65 (1990) 964 [INSPIRE].

[60] M.E. Peskin and T. Takeuchi, Estimation of oblique electroweak corrections, Phys. Rev. D 46 (1992) 381 [INSPIRE].

[61] K.S. Babu, C.F. Kolda and J. March-Russell, Implications of generalized Z-Z' mixing, Phys. Rev. D 57 (1998) 6788 [hep-ph/9710441] [INSPIRE].

[62] J. Kumar and J.D. Wells, CERN LHC and ILC probes of hidden-sector gauge bosons, Phys. Rev. D 74 (2006) 115017 [hep-ph/0606183] [INSPIRE].

[63] W.-F. Chang, J.N. Ng and J.M.S. Wu, A very narrow shadow extra Z-boson at colliders, Phys. Rev. D 74 (2006) 095005 [Erratum ibid. D 79 (2009) 039902] [hep-ph/0608068] [INSPIRE].

[64] C.-Y. Chen, S. Dawson and E. Furlan, Vector-like fermions and Higgs effective field theory revisited, arXiv: 1703.06134 [INSPIRE].

[65] GFitTer Group collaboration, M. Baak et al., The global electroweak fit at NNLO and prospects for the LHC and ILC, Eur. Phys. J. C 74 (2014) 3046 [arXiv:1407.3792] [INSPIRE]. 
[66] N. Kumar and S.P. Martin, Vectorlike leptons at the Large Hadron Collider, Phys. Rev. D 92 (2015) 115018 [arXiv:1510.03456] [INSPIRE].

[67] LEPSUSYWG, ALEPH, DELPHI, L3 and OPAL experiments, http://lepsusy.web.cern.ch/lepsusy/.

[68] LEPSUSYWG, ALEPH, DELPHI, L3 and OPAL experiments, Combined LEP chargino results, up to $208 \mathrm{GeV}$ for large $m_{0}$, LEPSUSYWG/01-03.1 (2001).

[69] P.J. Fox, R. Harnik, J. Kopp and Y. Tsai, LEP shines light on dark matter, Phys. Rev. D 84 (2011) 014028 [arXiv: 1103.0240] [INSPIRE].

[70] DELPHI collaboration, J. Abdallah et al., Photon events with missing energy in $e^{+} e^{-}$ collisions at $\sqrt{s}=130 \mathrm{GeV}$ to $209 \mathrm{GeV}$, Eur. Phys. J. C 38 (2005) 395 [hep-ex/0406019] [INSPIRE].

[71] M.R. Buckley, D. Feld and D. Goncalves, Scalar simplified models for dark matter, Phys. Rev. D 91 (2015) 015017 [arXiv: 1410.6497] [INSPIRE].

[72] P. Harris, V.V. Khoze, M. Spannowsky and C. Williams, Constraining dark sectors at colliders: beyond the effective theory approach, Phys. Rev. D 91 (2015) 055009 [arXiv:1411.0535] [INSPIRE].

[73] N. Craig, H.K. Lou, M. McCullough and A. Thalapillil, The Higgs portal above threshold, JHEP 02 (2016) 127 [arXiv: 1412.0258] [INSPIRE].

[74] CMS collaboration, Search for new physics in final states with two opposite-sign, same-flavor leptons, jets and missing transverse momentum in pp collisions at $\sqrt{s}=13 \mathrm{TeV}$, CMS-PAS-SUS-16-034 (2016).

[75] J. Alwall et al., The automated computation of tree-level and next-to-leading order differential cross sections and their matching to parton shower simulations, JHEP 07 (2014) 079 [arXiv: 1405.0301] [InSPIRE].

[76] A. Alloul, N.D. Christensen, C. Degrande, C. Duhr and B. Fuks, FeynRules 2.0 - A complete toolbox for tree-level phenomenology, Comput. Phys. Commun. 185 (2014) 2250 [arXiv: 1310.1921] [INSPIRE].

[77] CMS collaboration, Search for electroweak production of charginos and neutralinos in multilepton final states in pp collision data at $\sqrt{s}=13$ TeV, CMS-PAS-SUS-16-039 (2016).

[78] CMS collaboration, Search for new physics in events with two low momentum opposite-sign leptons and missing transverse energy at $\sqrt{s}=13 \mathrm{TeV}$, CMS-PAS-SUS-16-048 (2016).

[79] G.D. Kribs, T. Plehn, M. Spannowsky and T.M.P. Tait, Four generations and Higgs physics, Phys. Rev. D 76 (2007) 075016 [arXiv:0706.3718] [INSPIRE].

[80] M. Hashimoto, Constraints on mass spectrum of fourth generation fermions and Higgs bosons, Phys. Rev. D 81 (2010) 075023 [arXiv:1001.4335] [INSPIRE].

[81] K. Ishiwata and M.B. Wise, Higgs properties and fourth generation leptons, Phys. Rev. D 84 (2011) 055025 [arXiv:1107.1490] [INSPIRE].

[82] N. Arkani-Hamed, K. Blum, R.T. D'Agnolo and J. Fan, $2: 1$ for naturalness at the LHC?, JHEP 01 (2013) 149 [arXiv: 1207.4482] [INSPIRE].

[83] G. Isidori, G. Ridolfi and A. Strumia, On the metastability of the standard model vacuum, Nucl. Phys. B 609 (2001) 387 [hep-ph/0104016] [INSPIRE]. 
[84] D. Buttazzo et al., Investigating the near-criticality of the Higgs boson, JHEP 12 (2013) 089 [arXiv: 1307.3536] [INSPIRE].

[85] M.E. Machacek and M.T. Vaughn, Two loop renormalization group equations in a general quantum field theory. 1. Wave function renormalization, Nucl. Phys. B 222 (1983) 83 [INSPIRE].

[86] M.E. Machacek and M.T. Vaughn, Two loop renormalization group equations in a general quantum field theory. 2. Yukawa couplings, Nucl. Phys. B 236 (1984) 221 [InSPIRE].

[87] M.E. Machacek and M.T. Vaughn, Two loop renormalization group equations in a general quantum field theory. 3. Scalar quartic couplings, Nucl. Phys. B 249 (1985) 70 [inSPIRE].

[88] S.P. Martin, Top-quark pole mass in the tadpole-free $\overline{M S}$ scheme, Phys. Rev. D 93 (2016) 094017 [arXiv: 1604.01134] [INSPIRE].

[89] J.M. Cline, G. Dupuis, Z. Liu and W. Xue, The windows for kinetically mixed Z'-mediated dark matter and the galactic center gamma ray excess, JHEP 08 (2014) 131

[arXiv:1405.7691] [INSPIRE].

[90] H. Baer, K.-m. Cheung and J.F. Gunion, A heavy gluino as the lightest supersymmetric particle, Phys. Rev. D 59 (1999) 075002 [hep-ph/9806361] [INSPIRE].

[91] M. Lattanzi and J.I. Silk, Can the WIMP annihilation boost factor be boosted by the Sommerfeld enhancement?, Phys. Rev. D 79 (2009) 083523 [arXiv:0812.0360] [INSPIRE].

[92] A. Falkowski, J. Juknevich and J. Shelton, Dark matter through the neutrino portal, arXiv:0908.1790 [INSPIRE].

[93] G. Jungman, M. Kamionkowski and K. Griest, Supersymmetric dark matter, Phys. Rept. 267 (1996) 195 [hep-ph/9506380] [INSPIRE].

[94] J. Halverson, N. Orlofsky and A. Pierce, Vectorlike leptons as the tip of the dark matter iceberg, Phys. Rev. D 90 (2014) 015002 [arXiv: 1403.1592] [INSPIRE].

[95] R.J. Hill and M.P. Solon, Standard model anatomy of WIMP dark matter direct detection II: QCD analysis and hadronic matrix elements, Phys. Rev. D 91 (2015) 043505 [arXiv: 1409.8290] [INSPIRE].

[96] F. Bishara, J. Brod, B. Grinstein and J. Zupan, Chiral effective theory of dark matter direct detection, JCAP 02 (2017) 009 [arXiv:1611.00368] [INSPIRE].

[97] P. Junnarkar and A. Walker-Loud, Scalar strange content of the nucleon from lattice QCD, Phys. Rev. D 87 (2013) 114510 [arXiv:1301.1114] [INSPIRE].

[98] PandaX-II collaboration, A. Tan et al., Dark matter results from first 98.7 days of data from the PandaX-II experiment, Phys. Rev. Lett. 117 (2016) 121303 [arXiv:1607.07400] [INSPIRE].

[99] LUX collaboration, D.S. Akerib et al., Results from a search for dark matter in the complete LUX exposure, Phys. Rev. Lett. 118 (2017) 021303 [arXiv: 1608. 07648] [INSPIRE].

[100] CDMS-II collaboration, Z. Ahmed et al., Dark matter search results from the CDMS II experiment, Science 327 (2010) 1619 [arXiv:0912.3592] [INSPIRE].

[101] G.B. Gelmini and P. Gondolo, Neutralino with the right cold dark matter abundance in (almost) any supersymmetric model, Phys. Rev. D 74 (2006) 023510 [hep-ph/0602230] [INSPIRE]. 
[102] D. Tucker-Smith and N. Weiner, Inelastic dark matter, Phys. Rev. D 64 (2001) 043502 [hep-ph/0101138] [INSPIRE].

[103] A.E. Faraggi and M. Pospelov, Selfinteracting dark matter from the hidden heterotic string sector, Astropart. Phys. 16 (2002) 451 [hep-ph/0008223] [INSPIRE].

[104] J.E. Juknevich, D. Melnikov and M.J. Strassler, A pure-glue hidden valley I. States and decays, JHEP 07 (2009) 055 [arXiv:0903.0883] [INSPIRE].

[105] K.K. Boddy, J.L. Feng, M. Kaplinghat and T.M.P. Tait, Self-interacting dark matter from a non-Abelian hidden sector, Phys. Rev. D 89 (2014) 115017 [arXiv: 1402.3629] [INSPIRE].

[106] K.K. Boddy, J.L. Feng, M. Kaplinghat, Y. Shadmi and T.M.P. Tait, Strongly interacting dark matter: Self-interactions and keV lines, Phys. Rev. D 90 (2014) 095016 [arXiv: 1408.6532] [INSPIRE].

[107] C.J. Morningstar and M.J. Peardon, The glueball spectrum from an anisotropic lattice study, Phys. Rev. D 60 (1999) 034509 [hep-lat/9901004] [INSPIRE].

[108] Y. Chen et al., Glueball spectrum and matrix elements on anisotropic lattices, Phys. Rev. D 73 (2006) 014516 [hep-lat/0510074] [INSPIRE].

[109] H.B. Meyer, Glueball matrix elements: a lattice calculation and applications, JHEP 01 (2009) 071 [arXiv: 0808.3151] [inSPIRE].

[110] N. Craig, A. Katz, M. Strassler and R. Sundrum, Naturalness in the dark at the LHC, JHEP 07 (2015) 105 [arXiv: 1501.05310] [INSPIRE].

[111] D. Curtin and C.B. Verhaaren, Discovering uncolored naturalness in exotic Higgs decays, JHEP 12 (2015) 072 [arXiv: 1506. 06141] [INSPIRE].

[112] J.P. Chou, D. Curtin and H.J. Lubatti, New detectors to explore the lifetime frontier, Phys. Lett. B 767 (2017) 29 [arXiv: 1606.06298] [INSPIRE].

[113] P. Schwaller, D. Stolarski and A. Weiler, Emerging jets, JHEP 05 (2015) 059 [arXiv: 1502.05409] [INSPIRE].

[114] T. Cohen, M. Lisanti and H.K. Lou, Semivisible jets: dark matter undercover at the LHC, Phys. Rev. Lett. 115 (2015) 171804 [arXiv:1503.00009] [INSPIRE].

[115] M. Baumgart, C. Cheung, J.T. Ruderman, L.-T. Wang and I. Yavin, Non-Abelian dark sectors and their collider signatures, JHEP 04 (2009) 014 [arXiv:0901.0283] [INSPIRE].

[116] J. Choquette and J.M. Cline, Minimal non-Abelian model of atomic dark matter, Phys. Rev. D 92 (2015) 115011 [arXiv: 1509.05764] [INSPIRE].

[117] G. Barello, S. Chang and C.A. Newby, Correlated signals at the energy and intensity frontiers from non-Abelian kinetic mixing, Phys. Rev. D 94 (2016) 055018 [arXiv: 1511.02865] [INSPIRE].

[118] L.B. Okun, Thetons, JETP Lett. 31 (1980) 144 [Pisma Zh. Eksp. Teor. Fiz. 31 (1979) 156] [INSPIRE].

[119] L.B. Okun, Theta particles, Nucl. Phys. B 173 (1980) 1 [InSPIRE].

[120] J. Kang and M.A. Luty, Macroscopic strings and 'quirks' at colliders, JHEP 11 (2009) 065 [arXiv:0805.4642] [INSPIRE].

[121] S.P. Martin, Quirks in supersymmetry with gauge coupling unification, Phys. Rev. D 83 (2011) 035019 [arXiv:1012.2072] [INSPIRE]. 
[122] R. Harnik, G.D. Kribs and A. Martin, Quirks at the Tevatron and beyond, Phys. Rev. D 84 (2011) 035029 [arXiv: 1106.2569] [INSPIRE].

[123] K. Cheung, W.-Y. Keung and T.-C. Yuan, Phenomenology of iquarkonium, Nucl. Phys. B 811 (2009) 274 [arXiv:0810.1524] [INSPIRE].

[124] V.D. Barger et al., Superheavy quarkonium production and decays: a new Higgs signal, Phys. Rev. D 35 (1987) 3366 [Erratum ibid. D 38 (1988) 1632] [INSPIRE].

[125] Z. Chacko, D. Curtin and C.B. Verhaaren, A quirky probe of neutral naturalness, Phys. Rev. D 94 (2016) 011504 [arXiv: 1512.05782] [INSPIRE].

[126] C. Jacoby and S. Nussinov, The relic abundance of massive colored particles after a late hadronic annihilation stage, arXiv:0712.2681 [INSPIRE].

[127] S. Nussinov and C. Jacoby, Some comments on the 'quirks' scenario, arXiv:0907.4932 [INSPIRE].

[128] M. Kawasaki, K. Kohri and T. Moroi, Big-Bang nucleosynthesis and hadronic decay of long-lived massive particles, Phys. Rev. D 71 (2005) 083502 [astro-ph/0408426] [INSPIRE].

[129] K. Jedamzik, Big Bang nucleosynthesis constraints on hadronically and electromagnetically decaying relic neutral particles, Phys. Rev. D 74 (2006) 103509 [hep-ph/0604251] [INSPIRE].

[130] X.-L. Chen and M. Kamionkowski, Particle decays during the cosmic dark ages, Phys. Rev. D 70 (2004) 043502 [astro-ph/0310473] [INSPIRE].

[131] A. Fradette, M. Pospelov, J. Pradler and A. Ritz, Cosmological constraints on very dark photons, Phys. Rev. D 90 (2014) 035022 [arXiv: 1407.0993] [InSPIRE].

[132] I. Garcia Garcia, R. Lasenby and J. March-Russell, Twin Higgs WIMP dark matter, Phys. Rev. D 92 (2015) 055034 [arXiv: 1505.07109] [INSPIRE].

[133] L. Forestell, D.E. Morrissey and K. Sigurdson, Non-Abelian dark forces and the relic densities of dark glueballs, Phys. Rev. D 95 (2017) 015032 [arXiv: 1605.08048] [INSPIRE].

[134] A. Soni, H. Xiao and Y. Zhang, A cosmic selection rule for glueball dark matter relic density, arXiv: 1704.02347 [INSPIRE].

[135] M.B. Voloshin, CP violation in Higgs diphoton decay in models with vectorlike heavy fermions, Phys. Rev. D 86 (2012) 093016 [arXiv:1208.4303] [INSPIRE].

[136] G. Passarino and M.J.G. Veltman, One loop corrections for $e^{+} e^{-}$annihilation into $\mu^{+} \mu^{-}$ in the Weinberg model, Nucl. Phys. B 160 (1979) 151 [INSPIRE]. 